\title{
Migrating Jupiter up to the habitable zone: Earth-like planet formation and water delivery
}

\author{
L. A. Darriba ${ }^{1,2}$, G. C. de Elía ${ }^{1,2}$, O. M. Guilera ${ }^{1,2}$, and A. Brunini ${ }^{3}$ \\ 1 Instituto de Astrofísica de La Plata, CCT La Plata-CONICET-UNLP, Paseo del Bosque S/N, 1900 La Plata, Argentina \\ e-mail: ldarriba@fcaglp.unlp.edu.ar \\ 2 Facultad de Ciencias Astronómicas y Geofísicas, Universidad Nacional de La Plata, Paseo del Bosque S/N, 1900 La Plata, \\ Argentina \\ 3 Universidad Nacional de La Patagonia Austral, Unidad Académica Caleta Olivia, Ruta 3 Acceso Norte, Caleta Olivia 9311, \\ Santa Cruz, CONICET, Argentina
}

Received 2 December 2016 / Accepted 22 July 2017

\begin{abstract}
Context. Several observational works have shown the existence of Jupiter-mass planets covering a wide range of semi-major axes around Sun-like stars.

Aims. We aim to analyse the planetary formation processes around Sun-like stars that host a Jupiter-mass planet at intermediate distances ranging from $\sim 1$ au to 2 au. Our study focusses on the formation and evolution of terrestrial-like planets and water delivery in the habitable zone (HZ) of the system. Our goal is also to analyse the long-term dynamical stability of the resulting systems. Methods. A semi-analytic model was used to define the properties of a protoplanetary disk that produces a Jupiter-mass planet around the snow line, which is located at $\sim 2.7$ au for a solar-mass star. Then, it was used to describe the evolution of embryos and planetesimals during the gaseous phase up to the formation of the Jupiter-mass planet, and we used the results as the initial conditions to carry out $N$-body simulations of planetary accretion. We developed sixty $N$-body simulations to describe the dynamical processes involved during and after the migration of the gas giant.

Results. Our simulations produce three different classes of planets in the HZ: "water worlds", with masses between $2.75 M_{\oplus}$ and $3.57 M_{\oplus}$ and water contents of $58 \%$ and $75 \%$ by mass, terrestrial-like planets, with masses ranging from $0.58 M_{\oplus}$ to $3.8 M_{\oplus}$ and water contents less than $1.2 \%$ by mass, and "dry worlds", simulations of which show no water. A relevant result suggests the efficient coexistence in the $\mathrm{HZ}$ of a Jupiter-mass planet and a terrestrial-like planet with a percentage of water by mass comparable to the Earth. Moreover, our study indicates that these planetary systems are dynamically stable for at least 1 Gyr.

Conclusions. Systems with a Jupiter-mass planet located at 1.5-2 au around solar-type stars are of astrobiological interest. These systems are likely to harbour terrestrial-like planets in the HZ with a wide diversity of water contents.
\end{abstract}

Key words. astrobiology - planets and satellites: dynamical evolution and stability - planets and satellites: formation planets and satellites: terrestrial planets - methods: numerical

\section{Introduction}

In recent years, observational works (Cumming et al. 2008; Howard 2013) and theoretical studies (Mordasini et al. 2009; Ida et al. 2013) have suggested the existence of a wide diversity of planetary architectures in the Universe. The number of confirmed planets discovered to date outside the solar system has grown to 3610, of which 1192 are associated with G-type stars. From this group, $\sim 25 \%$ are Jupiter-mass planets, covering a wide range of semi-major axes, which extend from 0.01 au to 6 au.

Gaseous giants are thought to be formed in the outer regions of a protoplanetary disk (e.g. Pollack et al. 1996). However, the observational evidence shows that a great number of giant exoplanets discovered around Sun-like stars are located at inner regions of the system, well inside the position of the so-called snowline at $\sim 3$ au. A peculiar kind of such "warm-giant planets" is represented by those orbiting within 0.1 au from the central star, which are known as "hot Jupiters". A possible explanation for the origin of such warm and hot Jupiters suggests that they formed by gradual accretion of solids and capture of gas at a larger distance from the star, and it subsequently migrated inwards through interactions with the gas disk, from a process known as "type II migration" (Lin et al. 1996).

Several authors have analysed the formation, evolution, and survival of terrestrial-like planets during and after gaseous giant migration. On the one hand, Armitage (2003) suggested a reduced efficiency of terrestrial-like planet formation following gas giant migration. In such a work, the author made use of a simple time dependent model for the evolution of gas and solids in the disk and inferred that replenishment of solid material in the inner disk, following the inward migration of a giant planet, is generally an inefficient process. From this, Armitage (2003) suggested that terrestrial planets are conceivable to survive in a modest fraction of systems in which a single generation of massive planets formed without significant migration. However, it is worth noting that the author did not consider the dynamical effects of the migrating giant on any planetesimals. On the other hand, Mandell \& Sigurdsson (2003) developed numerical simulations in order to analyse how the inward migration of a gas giant affects the evolution of inner terrestrial planets. To do this, the authors placed a planet identical to Jupiter at $5.2 \mathrm{au}$, which was allowed to migrate inward over different timescales through an inner terrestrial-like planet system. From this, they concluded 
that the inward migration of a giant planet does not invariably eliminate pre-formed terrestrial planets and that, given an initial layout of bodies similar to that of the solar system, between $1-4 \%$ of systems in which migration occurred could still possess a planet in the habitable zone (HZ). It is very important to take into account that none of these studies actually simulated the terrestrial planet formation process simultaneously with the giant planet migration.

Fogg \& Nelson (2005) developed the first work aimed at studying the inner system planetary accretion in the presence of a migrating giant. From $N$-body simulations, this study examined the oligarchic and giant-impact growth in a protoplanet or planetesimal disk extending between 0.4 au and 4 au. Five scenarios were considered, corresponding to five different ages for the inner planet forming disk at the point when a giant planet was assumed to form at 5 au and migrate in to $0.1 \mathrm{au}$. In all five of their scenarios, Fogg \& Nelson (2005) found that the majority of the disk solids survived the passage of the giant planet, either by being shepherded inward of the giant, or by being scattered by the giant into excited exterior orbits. Then, Fogg \& Nelson (2007) studied the formation of terrestrial-like planets in hotJupiter systems, including an improved model that incorporated a viscous evolving gas disk, annular gap and inner-cavity formation due to the gravitational field of the giant planet, and selfconsistent evolution of the giant's orbit. From this, the authors obtained that more than $60 \%$ of the embryos and planetesimals survives the migration of the giant planet by being scattered by it into external orbits, which results in the regeneration of a solid disk where it is possible for the formation process of terrestriallike planets to occur. Fogg \& Nelson (2007) inferred that the giant planet migration induces mixing of volatile-rich material from beyond the called snowline into the inner region of the disk, suggesting that hot-Jupiter systems are likely to harbour waterabundant terrestrial-like planets in their circumstellar HZs. It is worth remarking that in the works developed by Fogg \& Nelson $(2005,2007)$ the authors analysed the evolution of the disk and the formation process of terrestrial-like planets for a few Myr after the migration of the gaseous giant.

Mandell et al. (2007) carried out $N$-body simulations with the goal of exploring the formation of terrestrial-like planets during and after giant planet migration, analysing the dynamics of post-migration planetary systems over 200 Myr. These authors proposed models with a single migrating giant planet, as well as with one inner migrating and one outer non-migrating giant planet. This work suggests that the formation of terrestrial-like planets in the $\mathrm{HZ}$ seems to be a frequent process in such systems. However, an interesting result derived by Mandell et al. (2007) indicates that planets surviving in the $\mathrm{HZ}$ in systems with closein giant planets tend to accrete more water-rich material than those in systems with only outer gas giants.

Then, Fogg \& Nelson (2009) analysed the formation and evolution of terrestrial-like planets in warm-Jupiter systems. In particular, the authors studied the evolution of planetary systems in which a gaseous giant halts migration at semi-major axes in the range $0.13-1.7$ au due to the dissipation of the gas disk. A very interesting result derived by these authors suggests that terrestrial-like planets can survive in the HZ if the giant planet's final orbit lies outside the region of $\sim 0.3-1.5$ au for a $1 M_{\odot}$ star.

Here, we present results of numerical simulations aimed at analysing the effects generated by a migrating gaseous giant around a Sun-like star. In particular, our study focusses on the formation of terrestrial-like planets and water delivery in the $\mathrm{HZ}$ in systems in which a Jupiter-mass planet halts migration at semi-major axes in the range 1.3-2 au due to gas disk dispersal.
Among the works previously mentioned, that of Fogg \& Nelson (2009) is the most similar to that shown in this paper, though there are several relevant differences that deserve to be discussed. In fact, the main differences between the present paper and that carried out by Fogg \& Nelson (2009) are related to the mass of the protoplanetary disk, the size of the planetesimals, the physical and orbital properties of the giant planet, and the initial distributions of planetary embryos and planetesimals to be used in the $N$-body simulations. Moreover, unlike Fogg \& Nelson (2009), we propose to analyse the long-term dynamical stability of the resulting systems, integrating the simulations of interest for a time span of $1 \mathrm{Gyr}$. In particular, we believe our model improves the initial conditions assigned to embryos and planetesimals for the development of the $N$-body simulations in comparison with those proposed by Fogg \& Nelson (2009). This point of our research is very important because a more realistic treatment of the initial conditions associated to embryos and planetesimals will lead to a better determination of the physical and dynamical properties of the resulting planets. Moreover, our research offers a more detailed treatment concerning the long-term dynamical stability. This investigation will allow us to strengthen our knowledge concerning the astrobiological interest of planetary systems around Sun-like stars that host a Jupiter-mass planet at intermediate distances

This paper is therefore structured as follows. The properties of the protoplanetary disks used in our study are presented in Sect. 2. In Sect. 3, we discuss the semi-analytic model that allows us to describe the formation of the giant planet and the evolution of the system in the gas phase. Moreover, this model allows us to outline our choice of initial conditions for the $N$-body simulations. Then, we describe the main features of the $N$-body code used by us in Sect. 4. In Sect. 5, we show results and carry out a detailed analysis of all simulations. Finally, we carry out a discussion of these results and expose our conclusions in Sect. 6.

\section{Properties of the protoplanetary disk}

In order to determine the distribution of material in a protoplanetary disk, the most relevant parameter is the surface density. The surface density profiles of gas $\left(\Sigma_{\mathrm{g}}\right)$ and planetesimals $\left(\Sigma_{\mathrm{p}}\right)$ adopted in our model are the same as those corresponding to the classical minimum mass solar nebula (MMSN) derived by Hayashi (1981):

$$
\begin{aligned}
& \Sigma_{\mathrm{g}}(R)=1700\left(\frac{R}{1 \mathrm{au}}\right)^{-3 / 2} \mathrm{~g} \mathrm{~cm}^{-2}, \\
& \Sigma_{\mathrm{p}}(R)= \begin{cases}7.1\left(\frac{R}{1 \mathrm{au}}\right)^{-3 / 2} \mathrm{~g} \mathrm{~cm}^{-2}, & \text { if } R<2.7 \mathrm{au}, \\
30\left(\frac{R}{1 \mathrm{au}}\right)^{-3 / 2} \mathrm{~g} \mathrm{~cm}^{-2}, & \text { if } R>2.7 \mathrm{au},\end{cases}
\end{aligned}
$$

where $R$ represents the radial coordinate in the mid-plane, and the discontinuity at 2.7 au in the planetesimal surface density is caused by the condensation of volatiles, particularly the condensation of water (often called snow line).

In fact, for our work we have adopted a disk $N$ times more massive than the MMSN in order to trigger the formation of a giant planet just beyond the snow line. The numerical value of the scalar $N$ is accurately chosen using a semi-analytic model, which will be explained in detail in Sect. 3 .

In our model we have assumed a central solar-type star of $1 M_{\odot}$ and solar metallicity. Other important parameters, such as the mass of the protoplanetary disk $M_{\mathrm{d}}$, and the gas dissipation 
timescale $\tau$ must be quantified in order to narrow down the wide spectrum of possible scenarios for our simulations. Mamajek (2009) showed that the distribution of disk lifetimes decays exponentially with a timescale of $\sim 2.5 \mathrm{Myr}$, and Alexander et al. (2006) and Armitage (2010) showed that after a few Myr of viscous evolution, the disk is completely dissipated in a timescale of $\sim 10^{5} \mathrm{yr}$, when photoevaporation is included. Since the photoevaporation timescale is a factor $\sim 20$ smaller than the disk lifetime, in our work we have considered that the photoevaporation is instantaneous.

In order to define the values of $M_{\mathrm{d}}$ and $\tau$, it is necessary to develop a detailed study about the evolution of the protoplanetary disk during the gas phase with the aim of forming a giant planet beyond the snow line. In this case, we want to analyse the dynamical evolution of a planetary system with a gas giant migrating inward. In particular, we considered that the formation of the gaseous giant is a late process, so that its migration should be truncated at intermediate distances due to the dissipation of the gaseous disk.

To specify which protoplanetary disks can lead to this scenario, we used a semi-analytic model with which we were able to analyse the evolution of a planetary system in the gaseous phase. As we will see in the next section, this model allowed us to define the parameters $M_{\mathrm{d}}$, and $\tau$ as well as the initial conditions concerning the distribution of embryos and planetesimals to be used later in the $N$-body simulations.

\section{Semi-analytic model for the protoplanetary disk}

\subsection{Description}

Our model to calculate the formation of the planetary system and the giant planet is the one described in Guilera et al. (2010), Guilera et al. (2011) and Guilera et al. (2014). Here, we discuss the most important physical properties of the model, which calculates the formation of a planetary system immersed in a protoplanetary disk that evolves in time.

The protoplanetary disk is characterised by a gaseous and a solid component (a planetesimal disk). On the one hand, the gaseous component is dissipated exponentially,

$\Sigma_{\mathrm{g}}(t)=\Sigma_{\mathrm{g}}(t=0) \exp (-t / \tau)$

where $\tau$ is a characteristic timescale. On the other hand, the planetesimal disk evolves by planetesimal migration due to nebular drag and planetesimal accretion by the embryos (in this work we do not include the collisional evolution of the population of planetesimals). As a consequence of the mass conservation, the planetesimal surface density obeys a continuity equation,

$\frac{\partial \Sigma_{\mathrm{p}}\left(R, r_{\mathrm{p}}\right)}{\partial t}-\frac{1}{R} \frac{\partial}{\partial R}\left[R v_{\text {mig }}\left(R, r_{\mathrm{p}}\right) \Sigma_{\mathrm{p}}\left(R, r_{\mathrm{p}}\right)\right]=\mathcal{F}\left(R, r_{\mathrm{p}}\right)$,

where $r_{\mathrm{p}}$ represents the planetesimal radius, $v_{\text {mig }}$ the planetesimal migration velocity and $\mathcal{F}$ represents the sink terms due to the accretion by the embryos.

Two major processes govern the evolution of the eccentricities and inclinations of the planetesimal population along the disk:

- the gravitational stirring produced by the embryos (Ohtsuki et al. 2002);

- the damping due to nebular gas drag (Rafikov 2004; Chambers 2008).
The gas drag also causes an inward orbital migration of the planetesimals. The interaction between the planetesimals and the gas depends on the planetesimal relative velocities with respect to the gas, and on the ratio between the planetesimal radius and the gas molecular mean free path. As in Guilera et al. (2014), we considered three different regimes (Rafikov 2004; Chambers 2008):
- the Epstein regime;
- the Stokes regime;
- the quadratic regime.

Moreover, the embryos immersed in the disk grow by accretion of planetesimals in the oligarchic regime, and by the accretion of the surrounding gas. For the accretion of planetesimals, we used the prescriptions given by Inaba et al. (2001). Regarding the accretion of the surrounding gas, we solved the classical equation of transport and structure for the planet envelope (see Fortier et al. 2009; and Guilera et al. 2010, for detailed explanations). Finally, when the distance between two embryos becomes smaller than 3.5 mutual Hill radii, we considered the perfect merger between them. In this case, when two planets are merged we consider that the mass of the new planet is the sum of the core mass of the planets that are merged (we considered that the envelopes of the planets are dissipated), and the semi-major axis is the mean of the previous two semi-major axes (pondered by the mass of each planet). It is important to remark that in this work we have not considered type I migration for the planets. We considered the evolution of the planetary system taken into account only the in situ formation of the planets until one planet achieves the critical mass ${ }^{1}$ to become a giant planet. Regarding type I migration, its studies in idealised isothermal disks predict rapid inward migration rates (Tanaka et al. 2002), so it is necessary to reduce the migration rates using an ad-hoc factor to reproduce observations (Alibert et al. 2005; Ida \& Lin 2008; Miguel et al. 2011b,a). However, if more realistic disks are considered (Kley \& Crida 2008; Paardekooper et al. 2010, 2011) type I migration could substantially change. Moreover, when the mass of the planet becomes of the order of $10 M_{\oplus}$, corrotation torques become significant and type I migration is no longer linear. In this regime, migration is slowed or reversed (Masset et al. 2006; Dittkrist et al. 2014). But even in isothermal disks type I migration could be outward if full MHD turbulence is considered (Guilet et al. 2013). Finally, in a recent work, (Benítez-Llambay et al. 2015) found that if the released energy by the planet due to accretion of solid material is taken into account, this phenomena generates a heating torque which could significantly slow down, cancel, and even reverse inward type I migration for planets with masses $\lesssim 5 M_{\oplus}$. In light of these recent results, we consider our assumption of in situ formation could be considered as an approximation of a more complex problem.

\subsection{Application}

As we mentioned before, we applied our model to calculate the formation of a planetary system until one planet achieves the critical mass and becomes a giant planet. We applied the same methodology as in de Elía et al. (2013). We determined the mass of the disk to be used in our simulations calculating the in situ formation of an embryo located just beyond the snow line $(\sim 3 \mathrm{au})$ using our model of giant planet formation

\footnotetext{
1 As critical mass we considered the mass of the planet core when the mass of the envelope equals it and the planet starts the gaseous runaway growth.
} 


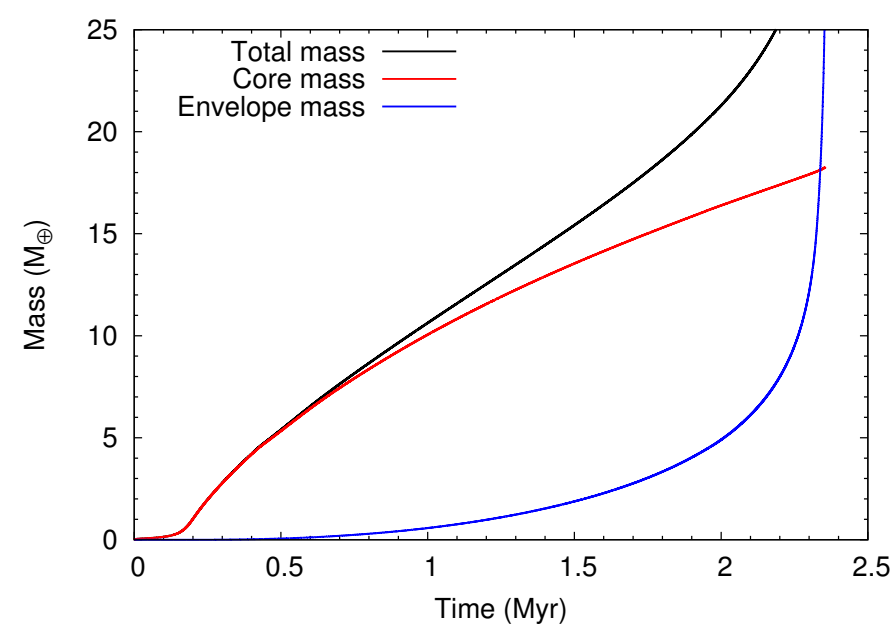

Fig. 1. Time evolution of the core mass, envelope mass, and total mass of a planet located just beyond the snow line $(\sim 3 \mathrm{au})$. Chosen a disk $\sim 1.35$ times more massive than the MMSN, and a characteristic timescale of $\tau=2.5 \mathrm{Myr}$ for the dissipation of the gas disk, a Jupiterlike planet is formed by the accretion of planetesimals with $100 \mathrm{~m}$ radius in 2.35 Myr.

(Guilera et al. 2010). In this work, we have considered a particular scenario wherein the formation of a giant planet is assumed to happen just before the dissipation of the disk. Chosen a characteristic timescale of $\tau=2.5 \mathrm{Myr}$ for the dissipation of the disk, and assuming instant photoevaporation, we find that our model allows us to form a Jupiter-like planet in 2.35 Myr using a disk $\sim 1.35$ times more massive than the MMSN, and using planetesimals with $100 \mathrm{~m}$ radius. This result is depicted in Fig. 1, where we show the time evolution of the masses from both the core and the envelope of a giant planet formed near the snow line $(\sim 3 \mathrm{au})$. We note that in this simulation we used grain opacities without a reduction respect to the interstellar values. However, Ormel (2014) and Mordasini (2014) found that grain opacities could be much lower than in the interstellar medium (ISM). Reducing the opacity due to grains to a value of $2 \%$ of the ISM values (Hubickyj et al. 2005) we found that, for the same disk, the cross-over mass is reached at $\sim 1 \mathrm{Myr}$ where the mass of the core at this time is $\sim 14 M_{\oplus}$. Another important assumption in our model of giant planet formation is that we did not consider a limitation in the accretion of gas. In order to analyse this assumption we performed a series of test-simulations using the code PlanetaLP (Ronco et al. 2017), where the limitation of the gas accretion onto the planet by the capability of the disk to supply enough material is considered, finding similar results if the Shakura-Sunyaev $\alpha$-parameter (Shakura \& Sunyaev 1973) takes values between $\alpha=10^{-2}-10^{-3}$. However, for small values of $\alpha$ $\left(\sim 10^{-4}\right)$ gas accretion becomes significantly different. We want to remark that these assumptions could lead to a possible different set of initial conditions for the $N$-body simulations (see below).

Once the mass of protoplanetary disk was specified, we analysed the evolution of embryos and planetesimals during the gaseous phase. In particular, our model evolves such populations inside and beyond the snow line, and the simulations stop when the giant planet is formed. With this approach, we obtain the picture of the system when the giant planet is already formed and there is still a small remnant of gas in the disk. To do this, we assumed that the initial mass of the embryos is the one corresponding to the beginning of the oligarchic growth regime (Ida \& Makino 1993). Moreover, as we mentioned above, we
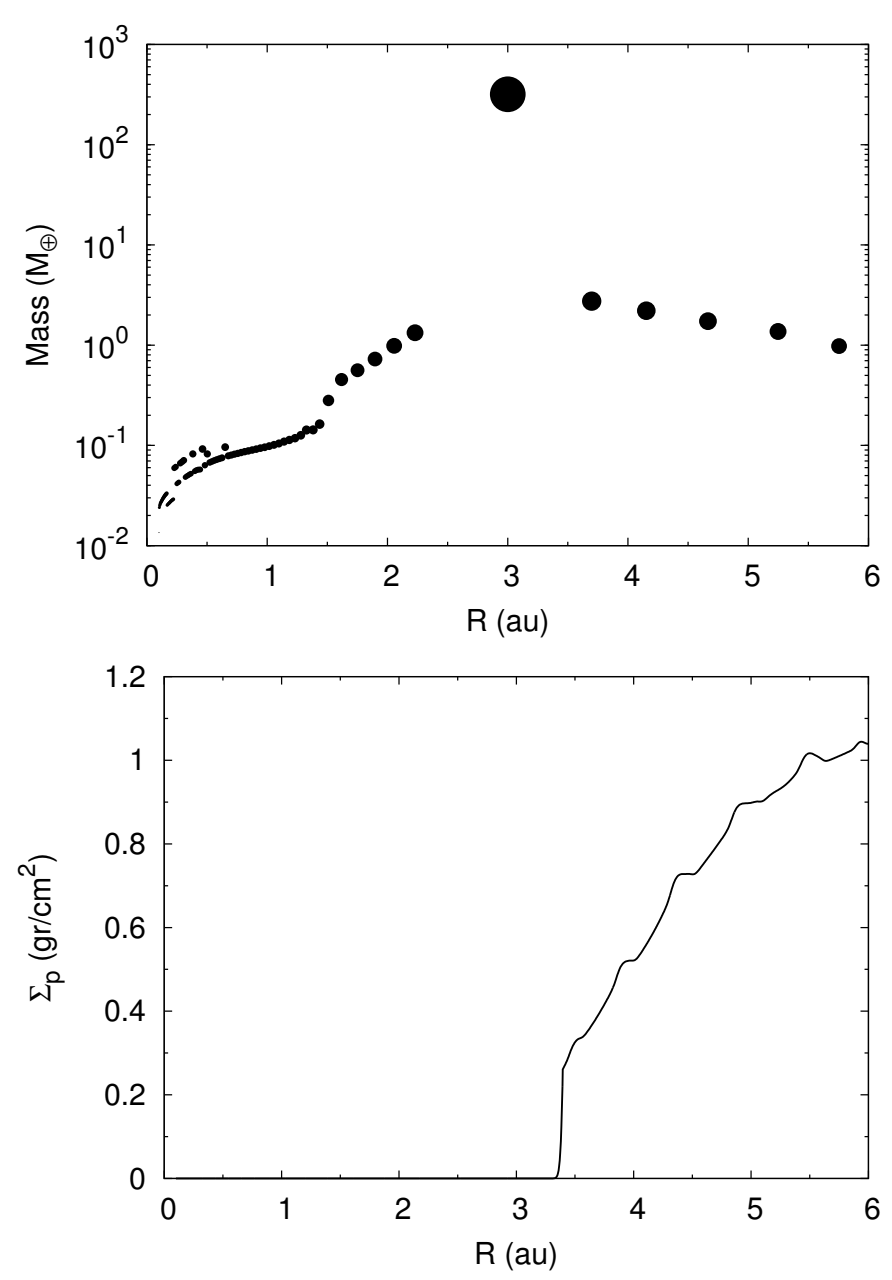

Fig. 2. Distribution of embryos (top) and planetesimals (bottom) when the Jupiter-like planet is formed. The point sizes represent the embryos scaled in mass. We can see that the planetary embryos and the gas giant accreted all the planetesimals inside $\sim 3.5$ au.

considered planetesimals with $100 \mathrm{~m}$ radius and a characteristic timescale of $\tau=2.5 \mathrm{Myr}$ for the dissipation of the disk. Since the timescale for collisions between planetesimals of $100 \mathrm{~m}$ radius is smaller than the accretion timescale when the embryos are relatively small, we neglected the collisional evolution of planetesimals. Figure 2 shows the distribution of embryos and planetesimals when the giant planet is formed. In particular, the top panel represents the mass distribution of planetary embryos as a function of the distance from the central star, while the bottom panel shows the surface density profile of planetesimals. These final distributions are used as initial condition for the $N$-body simulations.

\section{4. $\mathbf{N}$-body model}

\subsection{Characterisation and initial conditions}

The $N$-body code used to carry out our study is that developed by Chambers (1999) and known as MERCURY. In particular, we use the hybrid integrator, which uses a second-order mixed variable symplectic algorithm to treat the interaction between objects with separations greater than 3 Hill radii, and a BulirschStöer method for resolving closer encounters.

As we described in Sect. 3.2, we assumed that the gaseous component of the protoplanetary disk is dissipated in a timescale of $2.5 \mathrm{Myr}$, while the formation of the giant planet is a late 
process occurring within 2.35 Myr. Thus, a small amount of gas remains on the disk for $1.5 \times 10^{5} \mathrm{yr}$. In the present study, we analysed three distinct work scenarios, in which the gas giant migrates up to different distances respect to the central star during the $1.5 \times 10^{5} \mathrm{yr}$ after its formation. These scenarios are defined as follows:

- Scenario 1: the Jupiter-mass planet migrates up to $\sim 2 \mathrm{au}$; - Scenario 2: the Jupiter-mass planet migrates up to $\sim 1.6 \mathrm{au}$;

- Scenario 3: the Jupiter-mass planet migrates up to $\sim 1.3 \mathrm{au}$.

According to Tanaka \& Ida (1999), a giant planet can act as a shepherd, when its migration timescale is greater than some critical timescale, defined as a function of the gas dissipation timescale, or as a predator, in the other case. By doing the calculations, we find that all the giants on the simulations are predators.

In order to make use of the MERCURY code, it is mandatory to specify physical and orbital parameters for the gas giant planet, the planetary embryos, and the planetesimals. In all three scenarios, we assumed one Jupiter-mass planet with a physical density of $1.3 \mathrm{~g} \mathrm{~cm}^{-3}$. Immediately after formation, the giant planet might have a radius a factor of two larger than its final state, thus increasing the collisional cross section. However, we considered that this increase would not alter the dynamics of the system significantly. This planet is assigned a circular and planar orbit (i.e. $e=0$ and $i=0$ ) with a semi-major axis of 3 au. Moreover, the longitude of the ascending node $\Omega$, the argument of the pericentre $\omega$, and the mean anomaly $M$ are randomly generated in the range $\left[0,360^{\circ}\right]$.

We consider 41 planetary embryos between 0.5 au and 6 au in the three work scenarios. As we can see in the top panel of Fig. 2, the embryos have masses between $0.0135 M_{\oplus}$ and $2.75 M_{\oplus}$. For all planetary embryos, we assumed a physical density of $3 \mathrm{~g} \mathrm{~cm}^{-3}$. Regarding the orbital parameters, eccentricities and inclinations are randomly generated in the range $[0,0.02]$ and $\left[0,0.5^{\circ}\right]$, respectively. As for the giant planet, the longitude of the ascending node $\Omega$, the argument of the pericentre $\omega$ and the mean anomaly $M$ are randomly generated in the range $\left[0,360^{\circ}\right]$.

Finally, we consider 500 "super planetesimals", each one with a mass of $4.5 \times 10^{-3} \quad M_{\oplus}$ and a physical density of $1.5 \mathrm{~g} \mathrm{~cm}^{-3}$. The semi-major axes of the super planetesimals are generated using the acceptance-rejection method developed by John von Neumann, taking into account the surface density profile of planetesimals shown in the bottom panel of Fig. 2. Moreover, we assumed eccentricities and inclinations randomly generated in the range $[0,0.02]$ and $\left[0,0.5^{\circ}\right]$, respectively, and the values of $\Omega, \omega$ and $M$ are randomly generated in the range $\left[0,360^{\circ}\right]$ as in the case of embryos and the gas giant. The initial values for eccentricities and inclinations, both for embryos and planetesimals, were selected according to several authors who carried out $N$-body simulations, such as Raymond et al. (2004, 2006, 2009), O’Brien et al. (2006), Walsh \& Morbidelli (2011), to cite a few.

The term super planetesimal is used to denote particles that represent a large number of small planetesimals. In these simulations, the Jupiter-mass planet and the planetary embryos feel the gravitational presence of all the particles in the system, whereas the super planetesimals feel the effects of the central star and the big bodies, but they are non self-interacting. This consideration is included to reduce the CPU time and to avoid that the high masses of the super planetesimals unrealistically auto-excite the disk.
We assumed that the protoplanetary disk presents a radial composition gradient. In fact, given that the solid-surface density has a jump past the snow line, we assumed that bodies beyond 2.7 au contain $75 \%$ water by mass, while bodies inside $2.7 \mathrm{au}$ do not have water. This water distribution is assigned to planetary embryos and planetesimals of our simulations based on their starting locations. An aspect to take into account is that the MERCURY code evolves the orbits of planetary embryos and planetesimals, allowing collisions to occur. In all cases, collisions are treated as inelastic mergers preserving the mass and the water content.

Our main area of interest is the formation of planets inside the HZ. This zone represents a region around the central star where a planet can retain liquid water on its surface. Kopparapu et al. (2013b,a) established inner and outer limits for the HZ around stars of different types. In particular, the authors define conservative inner and outer edges of the HZ, which are determined by loss of water and by the maximum greenhouse provided by a $\mathrm{CO}_{2}$ atmosphere, respectively. For a Sun-like star, Kopparapu et al. (2013b,a) compute a conservative estimate for the width of the HZ of 0.99-1.67 au. However, the authors also determine more optimistic limits for the HZ. For the inner edge, an optimistic estimate is based on the inference that Venus has not had liquid water on its surface for at least the past one billion years (Solomon \& Head 1991). For the outer edge, a more optimistic empirical limit can be estimated based on the observation that early Mars was warm enough for liquid water to flow on its surface (Pollack et al. 1987; Bibring et al. 2006). For a Sun-like star, Kopparapu et al. (2013b,a) compute an optimistic estimate for the width of the $\mathrm{HZ}$ of 0.75-1.77 au. Given these estimates, we assumed that a planet is in the $\mathrm{HZ}$ of the system and so can hold permanent liquid water on its surface if its whole orbit is contained inside the optimistic limits, that is, if it has a perihelion $q \geq 0.75$ au and an aphelion $Q \leq 1.77 \mathrm{au}$.

However, it seems too conservative to require that the perihelion and aphelion distances are both inside the $\mathrm{HZ}$ for a planet to be habitable. Williams \& Pollard (2002) showed that, provided that an ocean is present to act as a heat capacitor, it is primarily the time-averaged flux that affects the habitability over an eccentric orbit. Planets with high orbital eccentricities $(e>0.1)$ have higher average orbital flux, which may help eccentric planets near the outer edge of the HZ to maintain habitable conditions. We will take this criterion into account for cases in which the planet has an aphelion on the outer edge of the HZ.

As the reader can see in both panels of Fig. 2, we considered that our study region extends from 0.5 au to 6 au. Then, we set the step-size of the integration to six days, which is shorter than $1 / 20$ th of the orbital period of the innermost body in the simulation. We integrated each simulation for at least $200 \mathrm{Myr}$, which is a good choice as an upper limit for the formation timescale of the terrestrial planets of our solar system (Touboul et al. 2007; Dauphas \& Pourmand 2011; Jacobson et al. 2014). Given the stochastic nature of the accretion process, we carried out twenty different numerical simulations for each of the three work scenarios.

The original MERCURY code was modified in order to incorporate some effects acting on the different system components. Remember that a small amount of gas remains in the system during the first $1.5 \times 10^{5} \mathrm{yr}$ of evolution. From this, we considered the effects of the type II migration on the Jupitermass planet. Then, we incorporated the effects of damping over the planetary embryos due to the presence of the gas. Finally, we added the effects of drag and damping over the planetesimals 
due to the action of the gaseous component. The inclusion of such effects is described in the following.

\subsection{Giant planet migration}

In order to model the type II migration, we considered a very simple drag acceleration, as described in Chiang et al. (2002), assuming that the giant planet migrates with a constant velocity, given by the expression

$\boldsymbol{a}_{\mathrm{mig}}=-\frac{\boldsymbol{v}}{\tau_{\mathrm{mig}}}$,

where $v$ is the velocity vector of the body, and $\tau_{\text {mig }}=1.5 \times 10^{5} \mathrm{yr}$ is the migration time of the gaseous giant.

\subsection{Effects over planetary embryos: eccentricity and inclination damping}

The eccentricities $e$ and inclinations $i$ of planetary embryos are damped due to interactions with the gaseous disk. According to Cresswell \& Nelson (2008), the eccentricity and inclination damping times $t_{\mathrm{e}}^{\mathrm{d}}$ and $t_{\mathrm{i}}^{\mathrm{d}}$, respectively, are given by

$$
\begin{aligned}
t_{\mathrm{e}}^{\mathrm{d}}= & \frac{t_{\mathrm{wave}}}{0.780} \\
& \times\left[1-0.14\left(\frac{e}{H / R}\right)^{2}+0.06\left(\frac{e}{H / R}\right)^{3}+0.18\left(\frac{e}{H / R}\right)\left(\frac{i}{H / R}\right)^{2}\right], \\
t_{\mathrm{i}}^{\mathrm{d}}= & \frac{t_{\mathrm{wave}}}{0.544} \\
& \times\left[1-0.30\left(\frac{i}{H / R}\right)^{2}+0.24\left(\frac{i}{H / R}\right)^{3}+0.14\left(\frac{e}{H / R}\right)^{2}\left(\frac{i}{H / R}\right)\right],
\end{aligned}
$$

where $t_{\text {wave }}$ is given by

$t_{\text {wave }}=\frac{M_{\star}}{m_{\mathrm{e}}} \frac{M_{\star}}{\Sigma_{\mathrm{g}} a_{\mathrm{e}}^{2}}\left(\frac{H}{R}\right)^{4} \Omega^{-1}$,

being $M_{\star}$ the stellar mass, $m_{\mathrm{e}}$ and $a_{\mathrm{e}}$ the mass and semi-major axis of the planetary embryo, respectively, $\Sigma_{\mathrm{g}}$ the gas surface density, $\Omega$ the angular velocity of the unperturbed disk, and $H / R$ is the disk's scale height-to-radius ratio. The parameter $H$ is given by

$H=\left(\frac{K T R^{3}}{G M_{\star} m}\right)^{1 / 2}$,

being $m$ the mass of an hydrogen molecule, the radial temperature profile $T$ is $T=280 R^{-1 / 2}$ (where $R$ is given in au and $T$ in Kelvin) and $K$ the Boltzmann constant.

Thus, the accelerations experienced by the planetary embryos due to the gaseous disk are written by

$$
\begin{aligned}
& \boldsymbol{a}_{\mathrm{e}}^{\mathrm{d}}=-2 \frac{\boldsymbol{v} \cdot \boldsymbol{r}}{r^{2} t_{\mathrm{e}}^{\mathrm{d}}} \boldsymbol{r} \\
& \boldsymbol{a}_{\mathrm{i}}^{\mathrm{d}}=-\frac{v_{z}}{t_{\mathrm{i}}^{\mathrm{d}}} \hat{\boldsymbol{k}},
\end{aligned}
$$

where $t_{\mathrm{e}}^{\mathrm{d}}$ and $t_{\mathrm{i}}^{\mathrm{d}}$ are given by Eqs. (6), $\boldsymbol{r}$ and $\boldsymbol{v}$ are the position and velocity vectors of the embryo, respectively, and $\hat{\boldsymbol{k}}$ is the unity vector pointing in the direction perpendicular to the mid-plane.

\subsection{Gas effects over planetesimals: inward migration and eccentricity and inclination damping}

Regarding the gas effect over the super-planetesimals, they experience a drag from the gas disk, where we consider a defined physically realistic planetesimal size. For simplicity purposes, each planetesimal is considered as a sphere of radius $r_{\mathrm{p}}$ and density $\rho_{\mathrm{p}}$. The expression for the gas drag is given by

$\boldsymbol{a}_{\text {drag }}=-\frac{3 C_{\mathrm{D}} \rho_{\mathrm{g}}}{8 r_{\mathrm{p}} \rho_{\mathrm{p}}} v_{\text {rel }} \boldsymbol{v}_{\mathrm{rel}}$,

(Adachi et al. 1976) where $r_{\mathrm{p}}$ and $\rho_{\mathrm{p}}$ are the radius and density of each individual planetesimal, respectively, $C_{\mathrm{D}}$ is a constant, which we define as $C_{\mathrm{D}}=1, \boldsymbol{v}_{\mathrm{rel}}$ is the vector velocity of the planetesimals relative to the gas, and $\rho_{\mathrm{g}}$ is the volume gas density, given by

$\rho_{\mathrm{g}}=\rho_{0} \mathrm{e}^{-z^{2} / 2 H^{2}} \mathrm{e}^{-t / \tau}$,

where $z$ is the coordinate normal to the mid-plane, and $\rho_{0}$ a constant given by

$\rho_{0}=\frac{\Sigma_{\mathrm{g}}}{H \sqrt{2 \pi}}$,

being $H$ is the height scale defined by Eq. (8), and $\tau$ the gas dissipation timescale specified in Sect. 2. The value chosen for $r_{\mathrm{p}}$ is $100 \mathrm{~m}$ (see Sect. 3.2). The planetesimals therefore experience both an orbital damping effect and an inward migration, decreasing with mass and increasing with gas density.

\section{Results}

In this section we show the results of the simulations corresponding to the three scenarios mentioned before. In particular, we are interested in analysing the formation of terrestrial planets in the $\mathrm{HZ}$, focussing on their final masses, water contents, and longterm dynamical stability.

\subsection{Scenario 1}

Here, we analyse the dynamical evolution of the systems in which the Jupiter-mass planet migrates up to $\sim 2$ au from the central star. This set of runs represents a complement of the work developed by Fogg \& Nelson (2009), who analysed the terrestriallike planet formation in systems in which a giant planet halts migration at semi-major axes in the range 0.13-1.7 au. In this Scenario 1 , six of the twenty $N$-body simulations form planets in the HZ of the system. In Table 1 we have listed the simulations which end with a planet in the HZ after $200 \mathrm{Myr}$ of evolution. For each planet, we detailed the semi-major axis at the beginning and at the end of the integration, together with the final mass and the final percentage of water by mass. As the reader can see, the planets formed in the HZ are super-Earths with a very wide range of final water contents.

The dynamical evolution of the six simulations that produce planets in the HZ is very similar. Thus, we analysed the results of one of them as a representative of the whole group. The simulation proposed is that labelled as Simulation 16, and is shown in Fig. 3. In each panel of this figure we can see snapshots at different times of the evolution on the semi-major axis-eccentricity plane. In Fig. 3, both embryos and planetesimals are quickly excited, reaching eccentricities of $e \sim 0.5$ in $1 \mathrm{Myr}$, together with the ejection of $\sim 40 \%$ of the planetesimals. At $10 \mathrm{Myr}, \sim 75 \%$ of 

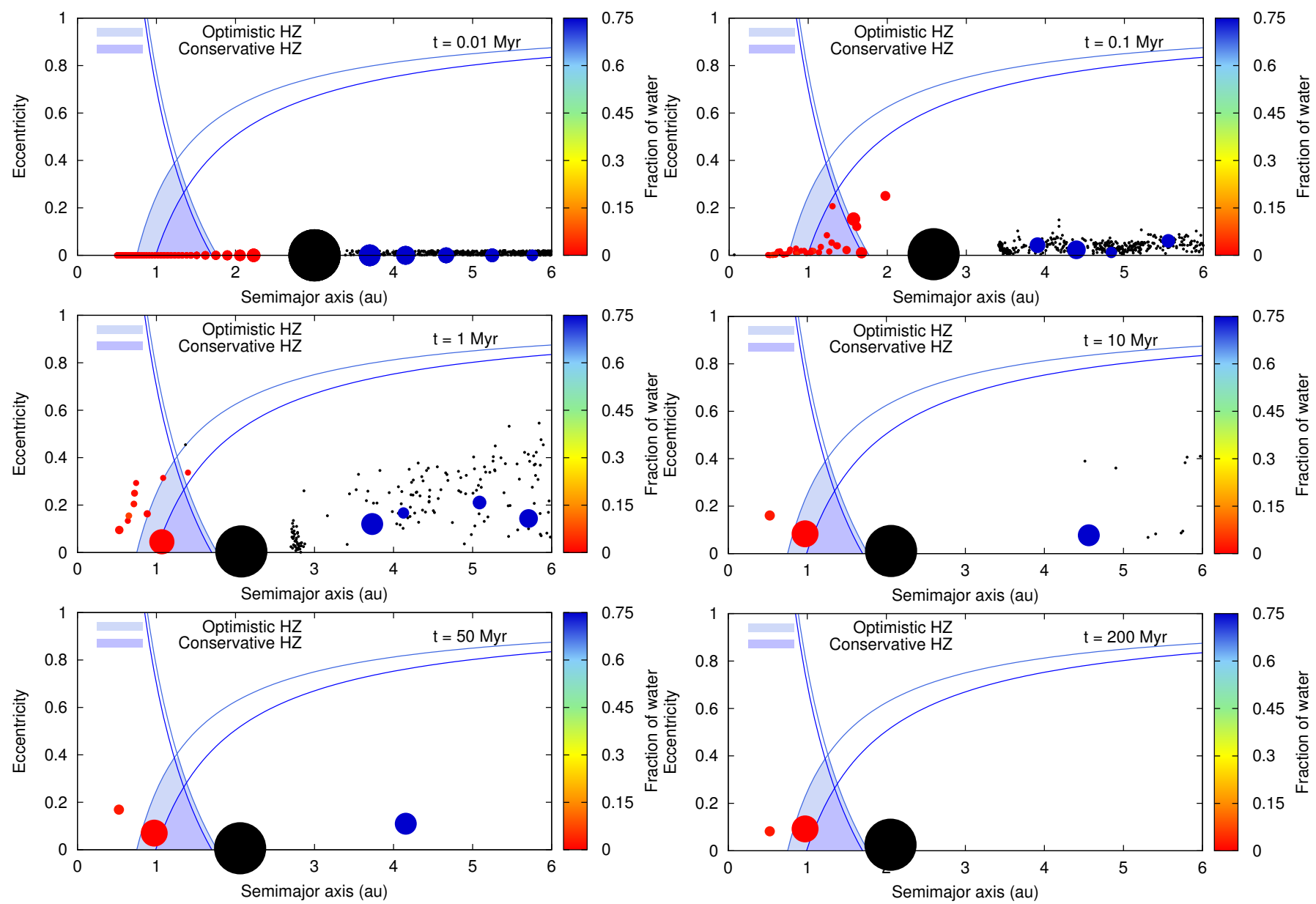

Fig. 3. Snapshots of the dynamical evolution for Simulation 16 of Scenario 1 . The blue and light-blue shaded areas represent the conservative and the optimistic HZ, respectively. In the same way, the blue and light-blue curves represent curves of constant perihelion and aphelion, both for the conservative and the optimistic HZ. The Jupiter-mass planet is plotted as a big black circle, the planetary embryos as coloured circles, and the planetesimals as small black points. The colour scale represents the fraction of water of the embryos respect to their total masses.

Table 1. Planets surviving in the $\mathrm{HZ}$ after $200 \mathrm{Myr}$ of evolution for Scenario 1.

\begin{tabular}{ccccc}
\hline \hline Simulation & $a_{\mathrm{i}}(\mathrm{au})$ & $a_{\mathrm{f}}(\mathrm{au})$ & $M\left(M_{\oplus}\right)$ & $W(\%)$ \\
\hline 3 & 2.23 & 0.90 & 2.15 & 0.00 \\
7 & 2.06 & 0.95 & 3.85 & 0.53 \\
11 & 2.23 & 1.09 & 2.40 & 0.43 \\
14 & 3.70 & 1.10 & 2.75 & 75.00 \\
16 & 2.23 & 0.97 & 3.62 & 0.09 \\
17 & 3.70 & 1.07 & 3.57 & 58.05 \\
\hline
\end{tabular}

Notes. $a_{\mathrm{i}}$ and $a_{\mathrm{f}}$ represent the initial and final semi-major axes in au, respectively, $M$ the final mass in $M_{\oplus}$, and $W$ the final percentage of water by mass after 200 Myr of evolution.

planetesimals are ejected, some embryos grow accreting other embryos, and others collide with the giant, leaving three superEarths, with masses ranging from $1 M_{\oplus}$ to $3 M_{\oplus}$. One of these planets is located inside the HZ, and remains there until the end of the integration. In fact, the embryos distribution barely change in the rest of the evolution. At $50 \mathrm{Myr}$, only 5\% of planetesimals remain, and they are outside our interest zone. Finally, between $50 \mathrm{Myr}$ and $200 \mathrm{Myr}$, the furthest embryo was pushed beyond 6 au. Thus, at the end of the simulation, only two planets remain in our area of study together with the gas giant. The innermost planet has a mass of $0.8 M_{\oplus}$ and the one inside the
HZ has a mass of $3.62 M_{\oplus}$, with a water content of $\sim 0.09 \%$ by mass, which corresponds to $\sim 12$ Earth oceans ${ }^{2}$. We also see that there is a near complete ejection of the initial planetesimals. In fact, $\sim 86 \%$ of them are ejected from the system, while only $1 \%$ remain outside our study region.

If we look at the results shown in Table 1, we can see that all planets surviving in the $\mathrm{HZ}$ end up the simulation with semimajor axes very similar to that corresponding to the Earth (i.e. $0.9 \mathrm{au} \leq a \leq 1.1 \mathrm{au}$ ). In order to analyse the potential habitability of such planets, we studied their orbital evolution for 1 Gyr. This was done by computing the evolution in time of the orbit's innermost and outermost points (i.e. perihelion and aphelion) for each planet surviving in the HZ. Figure 4 shows the evolution in time of the perihelion $(q)$ and aphelion $(Q)$ of the Jupiter-mass planet and the planet surviving in the HZ for the six simulations of Scenario 1 shown in Table 1 during 1 Gyr. For each simulation, the perihelion $(q)$ and aphelion $(Q)$ of the giant planet, shown in Fig. 4, decrease during its migration, remaining nearly constant after it stops. The values of $q$ and $Q$ of the inner planet, which are also shown in Fig. 4, also decrease on this period, falling inside the HZ, which is delimited by the dashed horizontal lines.

2 The amount of water on Earth's surface is $2.8 \times 10^{-4} M_{\oplus}$, which represents 1 Earth oceans. On the other hand, Marty (2012) suggested that the current water content in Earth's mantle is $\sim 2 \times 10^{-3} M_{\oplus}$. From these studies, the current Earth might have a water content of about $0.1-$ $0.2 \%$ by mass. 

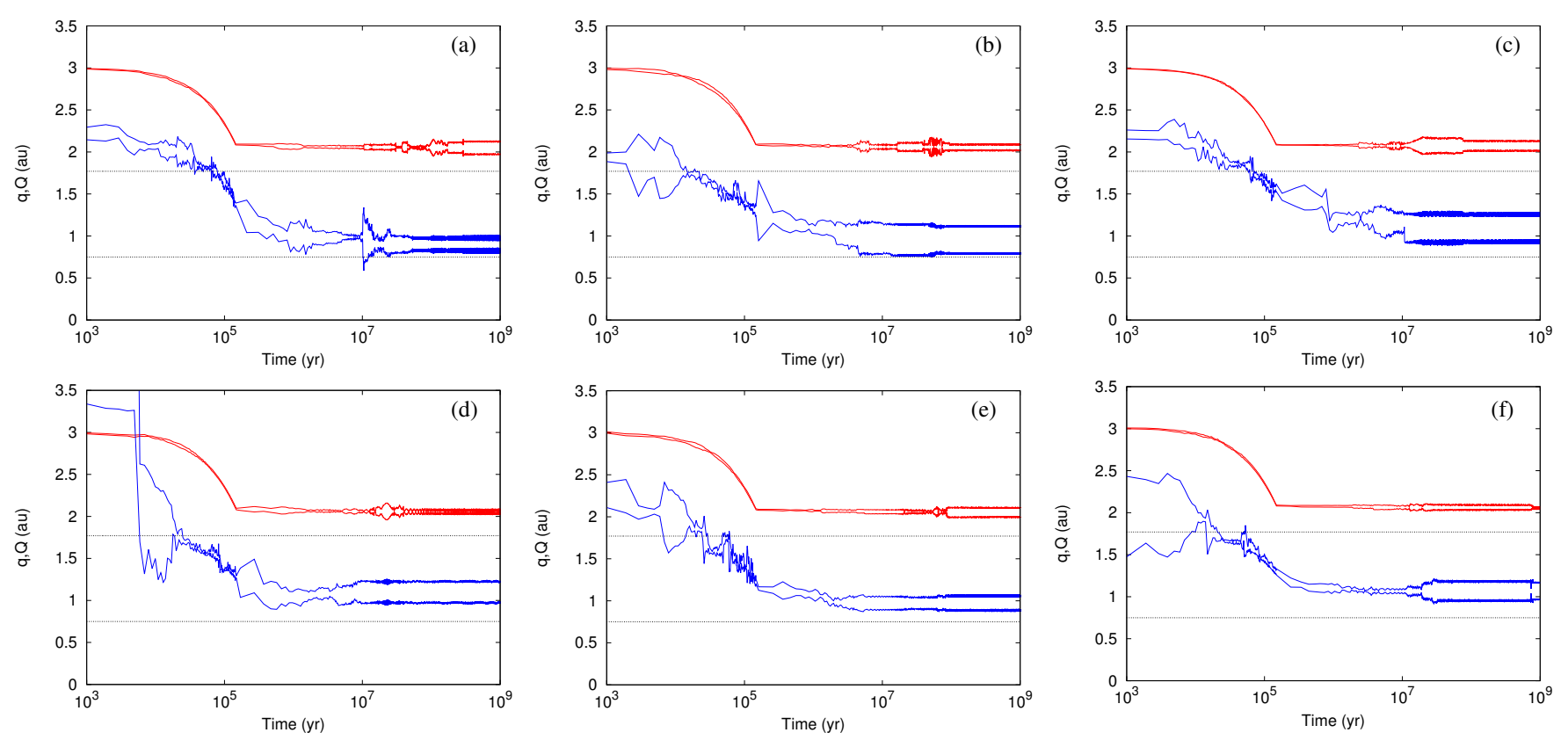

Fig. 4. Evolution in time of perihelion and aphelion for the Jupiter-mass planet (red curve) and the planet surviving in the HZ (blue curve). These results, obtained from Simulations 3, 7, 11, 14, 16, and 17 of Scenario 1, are shown in the panels a-f, respectively. The horizontal dashed lines represent the inner and outer edges of the optimistic HZ. These planetary configurations remain dynamically stable for $1 \mathrm{Gyr}$.

This indicates that the planet surviving in the HZ could retain liquid water on its surface, satisfying an essential requirement for the development and maintenance of life. We consider that this study suggests the long-term dynamical stability of the planets surviving in the HZ for Scenario 1.

Another relevant information shown in Table 1 is the mass of each planet surviving in the $\mathrm{HZ}$ after $200 \mathrm{Myr}$ of evolution. In all these simulations, the planets in the $\mathrm{HZ}$ are super-Earths with masses ranging from $2.15 M_{\oplus}$ to $3.85 M_{\oplus}$. Figure 5 shows the percentage of mass of each planet of Scenario 1 surviving in the $\mathrm{HZ}$ as a function of time. With the exception of the super-Earth formed in the Simulation 14, which does not collide with any other body during the entire integration, the rest of the planets acquire their final masses in less than 2 Myr. According to that suggested by Jacobson et al. (2014), this represents a timescale shorter than that associated to the formation of the Earth. In agreement with the generally accepted scenario, the last giant impact on Earth formed the Moon and initiated the final phase of core formation by melting Earth's mantle. Using highly siderophile element abundance measurements, Jacobson et al. (2014) determined a Moon-formation age of $95 \pm 32$ Myr after the condensation of the first solids in the solar system.

The last aspect we analyse is shown on the fifth column of Table 1, which represents the water content of each planet in the $\mathrm{HZ}$ at the end of the simulation. Our simulations produce three different kinds of planets in the HZ. The first kind is represented by planets whose accretion seed ${ }^{3}$ starts the simulation beyond the snow line. Such planets have significant primordial water contents and end up the simulation with very high water percentages by mass. Thus, they are called water worlds (see Kuchner 2003; and Léger et al. 2004, for instance, for further reference). The second kind of planets formed in the $\mathrm{HZ}$ is represented by those whose accretion seed starts the simulation inside the snow line and their feeding zones are primarily associated to 3 Following Raymond et al. (2009), we define a planet's accretion seed
as the larger body in each of its collisions.

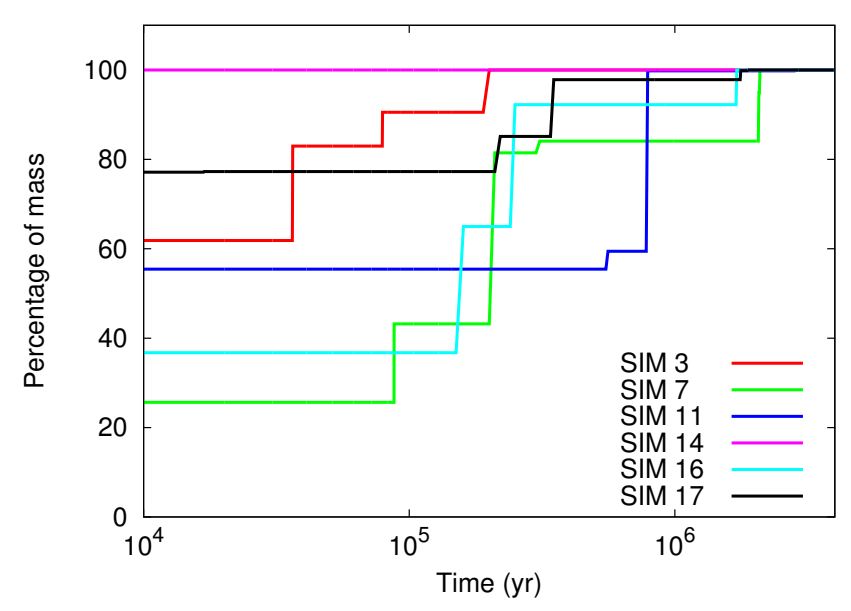

Fig. 5. Mass of the planets surviving in the HZ obtained from Scenario 1 as a function of time. All these planets have formation timescales less than $\sim 2$ Myr.

the inner region of the disk. Such planets end the simulation with water percentages less than $\lesssim 0.53 \%$ by mass. In general terms, the planets of this kind formed in our simulations are more massive than the Earth. However, such planets show features in their dynamical evolution and final water percentages that result to be comparable to the Earth. Thus, we decide to refer to this second kind of planets formed in the HZ of our simulations as "Earthlike planets". We infer that such planets have a very important astrobiological interest since they could achieve similar conditions to the Earth for the development and maintenance of life. Finally, the third kind of planets formed in the HZ is represented by those whose accretion seed starts the simulation inside the snow line and do not accrete water-rich material along the evolution. These planets end up the simulation with no water, and thus they are called dry worlds. 
In this scenario, we can see from Table 1 that the planets in the HZ associated to Simulations 14 and 17, which start their evolution at $a \sim 3.7 \mathrm{au}$, are truly water worlds. In fact, the planet in the $\mathrm{HZ}$ produced in Simulation 14 has a final mass of $2.75 M_{\oplus}$ and a final water content of $75 \%$, which equals to 7366 Earth oceans. In the same way, the planet in the HZ formed in Simulation 17 has a final mass of $3.57 M_{\oplus}$ and a final water content of $58 \%$, which equals to 7395 Earth oceans. The main difference between these two planets is that, since the one corresponding to Simulation 14 does not suffer any collision during its evolution, it preserves the initial water proportion. On the contrary, the planet in the HZ produced in Simulation 17 does experiment collisions with other bodies. In fact, this planet collides with three dry embryos, which contribute to the final mass, and with three water-rich planetesimals, which contribute to the water content. This phenomenon means that the planet in the HZ formed in Simulation 17 ends its evolution with a water percentage of $58 \%$ by mass. The final water contents of the water worlds formed in Scenario 1 are primarily determined by their primordial water contents. Thus, the water acquisition for such planets is an early process since it is associated to their formation.

On the other hand, the planets in the HZ associated to Simulations 7, 11 and 16 start the evolution with a semi-major axis inner to the snow line, so they do not contain initial water. These planets accrete water-rich planetesimals during their evolution, which play a primary role in such aspect. In fact, a significant result suggests that the planetesimals represent the main source of water of the Earth-like planets formed in Simulations 7, 11 and 16. Such as the Table 1 shows, the planet in the HZ formed in Simulation 7 has a final mass of $3.85 M_{\oplus}$ and a final water content of $0.53 \%$, which equals to $\sim 73$ Earth oceans. In the same way, the planet in the HZ formed in the Simulation 11 has a final mass of $2.4 M_{\oplus}$ and a final water content of $0.43 \%$, which equals to $\sim 37$ Earth oceans. Finally, the planet in the HZ associated to Simulation 16 has a final mass of $3.62 M_{\oplus}$ and a final water content of $0.09 \%$, which equals to $\sim 12$ Earth oceans. These three planets reach $75 \%$ of their final water contents in times of the order of their formation time. Thus, the water delivery on them is a late process in their evolutionary histories.

Finally, the planet in the HZ produced in Simulation 3 results to be a dry world. In fact, this planet ends the integration with no water, since it collides with planetary embryos formed inner to the snow line.

A relevant result derived from the present research indicates that a Jupiter-mass planet orbiting at $\sim 2$ au around a Sun-like star is able to coexist with super-Earths associated to the HZ with a wide diversity of water contents. It should be noted that, although none of the planets in the HZ is associated to mean motion resonances with the Jupiter-mass planet at the end of the simulations, there exist temporal captures of several embryos during its migration. For instance, in Simulation 7, an embryo with an initial semi-major axis of $\sim 1.43$ au is captured in a region near the 3:1 mean motion resonance with the Jupiter-mass planet up to $10^{3} \mathrm{yr}$. Another example is, in the same simulation, an embryo with an initial semi-major axis of $\sim 2.23 \mathrm{au}$, which is captured in the 3:2 mean motion resonance with the giant planet up to $\sim 5 \times 10^{4}$ yr. The captures into several mean motion resonances with the gas giant is a natural process in this kind of planetary systems.

\subsection{Scenario 2}

We studied the dynamical evolution of the systems in which the Jupiter-mass planet migrates up to $\sim 1.6$ au from the central star.
Table 2. Planets surviving in the $\mathrm{HZ}$ after $200 \mathrm{Myr}$ of evolution for Scenario 2.

\begin{tabular}{ccccc}
\hline \hline Simulation & $a_{\mathrm{i}}(\mathrm{au})$ & $a_{\mathrm{f}}(\mathrm{au})$ & $M\left(M_{\oplus}\right)$ & $W(\%)$ \\
\hline 1 & 0.50 & 0.94 & 0.58 & 1.18 \\
6 & 2.06 & 0.96 & 3.51 & 0.29 \\
14 & 2.23 & 0.82 & 3.50 & 0.10 \\
15 & 2.23 & 0.96 & 1.71 & 0.00 \\
\hline
\end{tabular}

Notes. $a_{\mathrm{i}}$ and $a_{\mathrm{f}}$ represent the initial and final semi-major axes in au, respectively, $M$ the final mass in $M_{\oplus}$, and $W$ the final percentage of water by mass after $200 \mathrm{Myr}$ of evolution.

In this particular scenario, four of the twenty $N$-body simulations produce planets in the $\mathrm{HZ}$ of the system after $200 \mathrm{Myr}$ of evolution. Table 2 shows the main properties of the planets surviving in the $\mathrm{HZ}$ at $200 \mathrm{Myr}$. For each of such planets, we have specified the semi-major axis at the beginning and at the end of the integration, as well as the final mass and the final percentage of water by mass.

Since all the simulations shown in Table 2 have an evolution which is dynamically comparable, we consider the one labelled " 6 " as a representative of the group to describe their evolution. Figure 6 shows snapshots in time on the semi-major axis-eccentricity plane of Simulation 6 , as we carried out in the previous section. The values of the time of each snapshot are the same as in Fig. 3. We can see from Fig. 6 that at $1 \mathrm{Myr}$, the embryos inner to the giant planet reach eccentricities $e \sim 0.25$, while the planetesimals are excited acquiring $e \leq 0.6$. This excitation is possibly due to the proximity of the gaseous giant, since it ends its migration inside the HZ. By 10 Myr more than 50\% of the planetesimals are ejected from the system. In addition, six planetary embryos remain in our interest zone. During the rest of the evolution, most planetesimals are ejected. At 50 Myr the system reaches a planetary configuration that remains without significant changes until the end of the evolution. At $200 \mathrm{Myr}$, the Jupiter-mass planet and a super-Earth of $3.51 M_{\oplus}$ with a water content of $0.29 \%$ by mass coexist in the HZ. We note that $\sim 75 \%$ of the initial planetesimals are ejected from the system, $\sim 6 \%$ remain further than our interest zone, and $\sim 6 \%$ with 0.5 au $\leq a \leq 6$ au.

In order to study the long-term dynamical stability of the planets surviving in the $\mathrm{HZ}$ at $200 \mathrm{Myr}$, we extend the four simulations listed in Table 2 for 1 Gyr. The planets associated to Simulations 1,6 , and 15 do not experience significant changes in their semi-major axes, eccentricities, an inclinations during 1 Gyr of evolution. These three simulations end up with a Jupiter-mass planet and a terrestrial-like planet inside the HZ. To ensure the potential habitability of such planets, we computed the evolution in time of their perihelia and aphelia, which are shown in Fig. 7. Our results indicate that the planets in the HZ associated to Simulations 1,6 , and 15 keep their perihelia and aphelia inside the $\mathrm{HZ}$ for at least $1 \mathrm{Gyr}$. This study helps us to conclude that such planets show a long-term dynamical stability and besides maintain habitability conditions for at least $1 \mathrm{Gyr}$ of evolution. However, the planet in the HZ formed in Simulation 14 shows differences in the long-term dynamical behaviour. In fact, such a planet has an encounter with another embryo at $\sim 920$ Myr that produces relevant changes in its semi-major axis. This dynamical event moves the perihelion of the planet outside the HZ, placing it at shorter distances from the central star than the inner edge of the optimistic HZ. Figure 8 shows the evolution of the perihelion and aphelion of the planet and the gas giant 

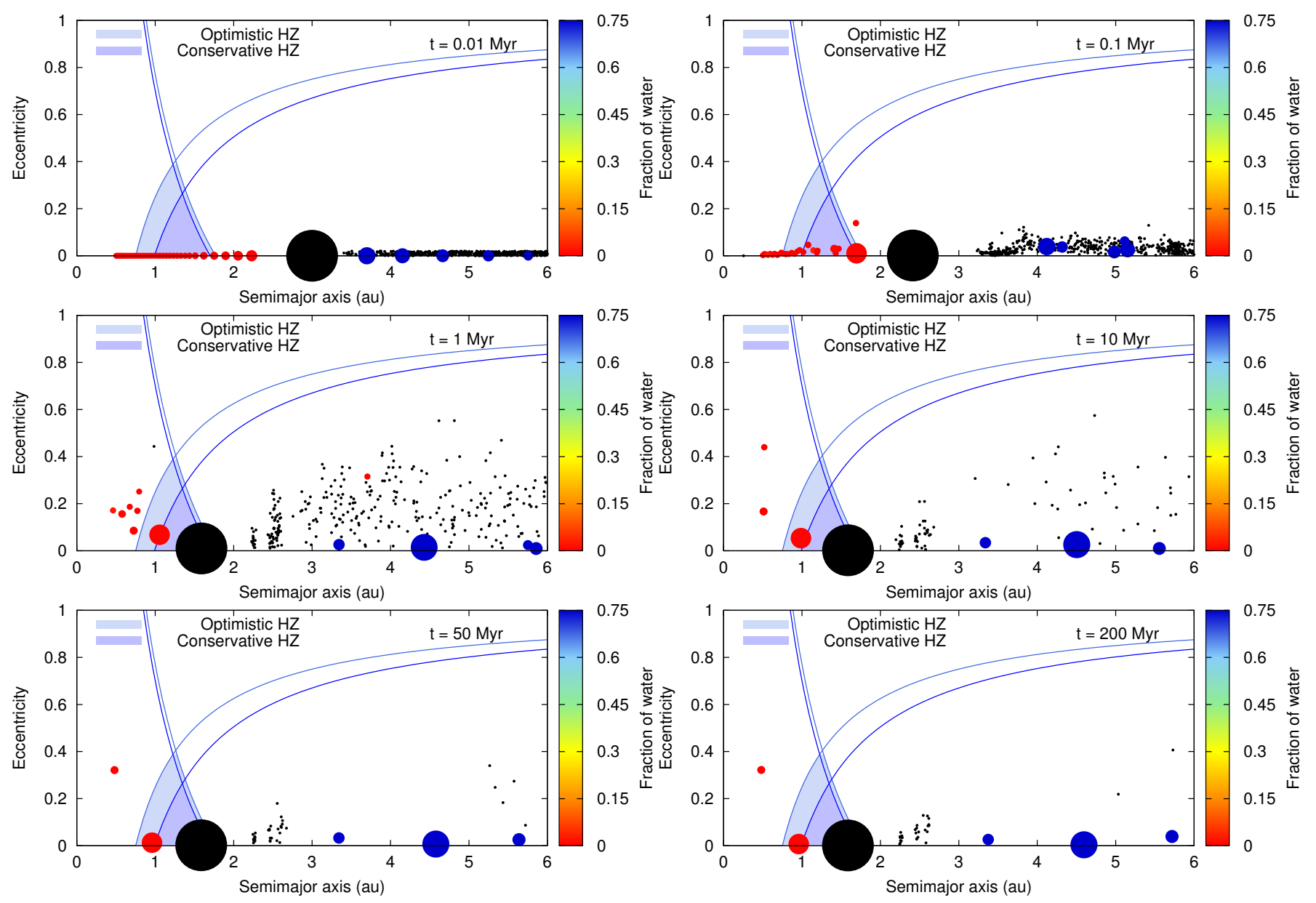

Fig. 6. Snapshots of the dynamical evolution for Simulation 6 of Scenario 2. The colour code description is analogous to that of Fig. 3.
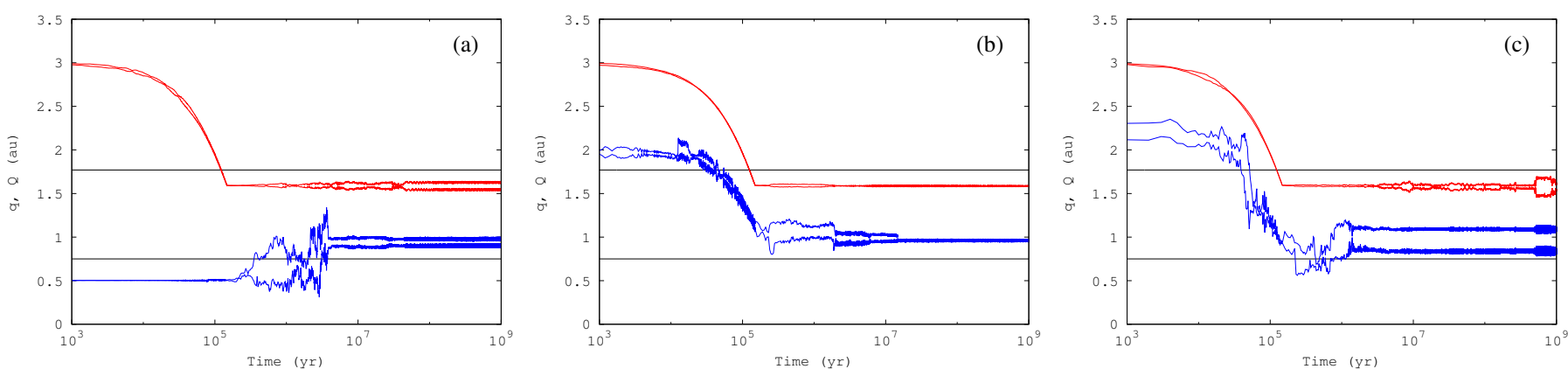

Fig. 7. Evolution in time of perihelion and aphelion for the Jupiter-mass planet (red curve) and the planet surviving in the HZ (blue curve). These results, obtained from Simulations 1, 6 and 15 of Scenario 2, are shown in the panels $a-c$, respectively. The horizontal dashed lines represent the inner and outer edges of the optimistic HZ. These planetary configurations remain dynamically stable for $1 \mathrm{Gyr}$.

as a function of the time. We can clearly see that the terrestriallike planet experiments a shift in its orbit nearly at the end of the integration. This phenomenon turns out to be crucial for the habitability, since a planet with such orbital properties can not retain liquid water on its surface. Thus, the late dynamical instability produced in Simulation 14 significantly modifies the orbit of the planet associated to the HZ, discarding it as potential target of astrobiological interest. Consequently, using dynamical criteria, the group of runs with potentially habitable planets is reduced to Simulations 1,6 and 15.

As Table 2 shows, the planets associated to the HZ in Simulations 1, 6, and 15 have masses of $0.58 M_{\oplus}, 3.51 M_{\oplus}$, and $1.71 M_{\oplus}$, respectively. Moreover, Figure 9 shows the percentage of mass of each of these planet surviving in the $\mathrm{HZ}$ as a function of time. This figure allows us to observe that the planets in the HZ resulting from Simulations 1, 6, and 15 acquiring $90 \%$ of their final mass at $1.6 \mathrm{Myr}, 1.7 \times 10^{4} \mathrm{yr}$, and $5 \times 10^{4} \mathrm{yr}$, respectively, which represent timescales shorter than that associated to the formation of the Earth (Jacobson et al. 2014). The planet in the HZ from Simulation 1 has the lowest mass of the three, with a final value of $0.58 \mathrm{M}_{\oplus}$. This difference from the other two simulations is also reflected in their initial masses, with the Simulation 1 mass being about one order of magnitude smaller. Its initial semi-major axis is also much smaller than the other two, being located near the internal border of the disk. All these features might contribute to the explanation that the planet of the 

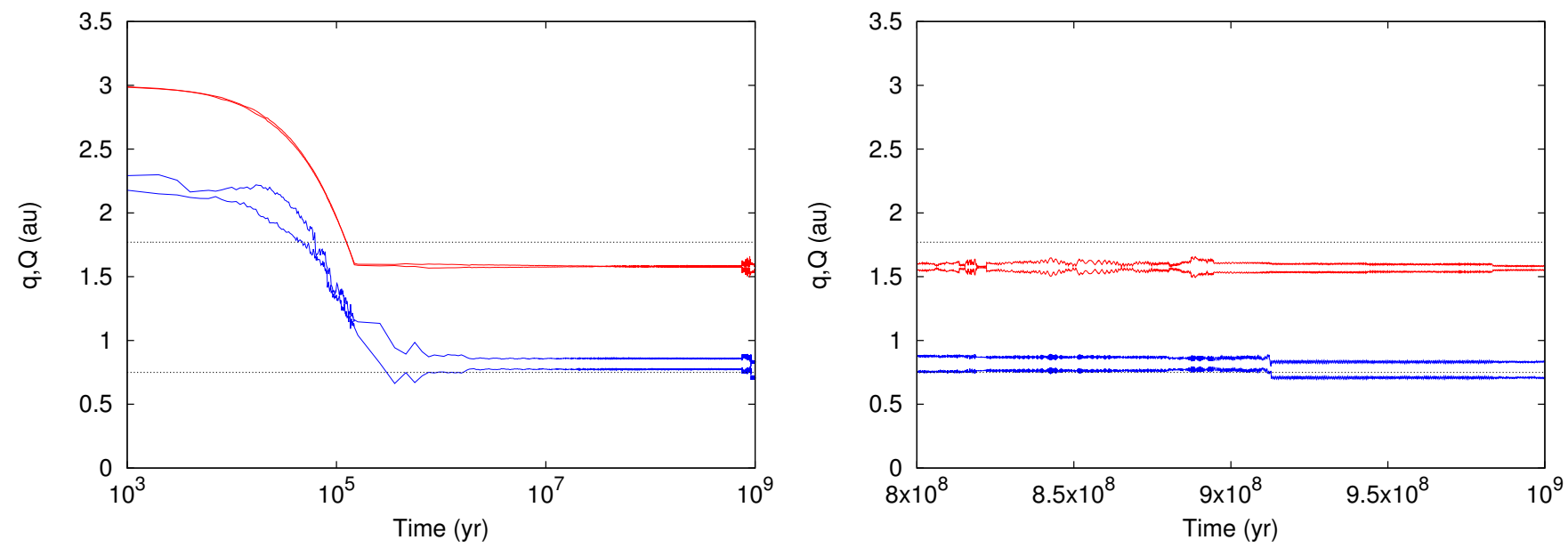

Fig. 8. Evolution in time of perihelion and aphelion for the Jupiter-mass planet (red curve) and the planet surviving in the HZ (blue curve). The results displayed on the left panel correspond to Simulation 14 of Scenario 2. The right panel shows a zoom at the last 200 Myr of evolution, where a significant encounter shift the perihelion of the planet out of the inner edge of the HZ.

$\mathrm{HZ}$ associated to Simulation 1 takes $\sim 10^{6}$ years to form, a time two orders of magnitude longer than the other two planets.

Regarding the water content, none of the planets in the $\mathrm{HZ}$ formed in Simulations 1, 6, and 15 is a water world. In fact, as Table 2 shows, these three planets start the simulation at distances from the central star closer than the snow line, so that they do not have primordial water contents. Moreover, Table 2 indicates that such planets end the simulation with percentages of water less than $\sim 1.2 \%$ by mass. In general terms, the three planets surviving in the $\mathrm{HZ}$ can be grouped into two different classes. On the one hand, the planet resulting from Simulation 15 is a dry world. In fact, this planet does not undergo collisions with water-rich material during its evolution, and thus it ends with no water at all. On the other hand, the planets in the HZ derived from Simulations 1 and 6 are Earth-like planets. In fact, as Table 2 shows, the planet resulting from Simulation 1 has a final mass of $0.58 M_{\oplus}$ and a final water content of $1.2 \%$ by mass, which is equal to $\sim 25$ Earth oceans. Our study indicates that this planet accretes its water content from the collision with an embryo at $\sim 1.6 \mathrm{Myr}$, which collided earlier with two outer waterrich planetesimals at $\sim 4 \times 10^{4} \mathrm{yr}$. In the same way, the planet in the $\mathrm{HZ}$ formed in Simulation 6 has a final mass of $3.51 M_{\oplus}$ and a final water content of $0.29 \%$ by mass, which is equal to $\sim 36$ Earth oceans. This planet acquires its water content by colliding with an embryo at $1.7 \times 10^{4} \mathrm{yr}$, which had earlier impacted with outer water-rich planetesimals. These planetesimals represent the main source of water for the planets in the HZ formed in Simulations 1 and 6. Moreover, by looking at their water accretion times, we can conclude that both planets undergo late water accretions with respect to their formation times.

As in Scenario 1, we also detect that several planetary embryos are temporarily captured in mean motion resonances with the Jupiter-mass planet. In particular, in Simulation 6 of the Scenario 2, the planet which ends in the HZ is captured in the 2:1 mean motion resonance with the gas giant during the evolution. This planet remains in this resonance for at least $1 \mathrm{Gyr}$.

\subsection{Scenario 3}

We analysed the dynamical evolution of the systems in which the Jupiter-mass planet migrates up to $\sim 1.3$ au from the central star. A main difference of this scenario with respect to the other

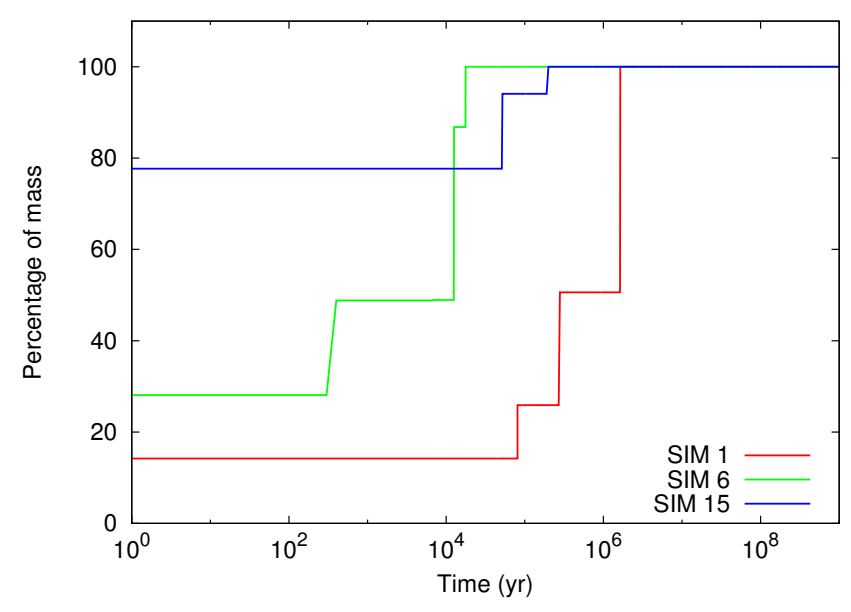

Fig. 9. Mass of the planets surviving in the HZ obtained from Scenario 2 as a function of time.

two is that none of the simulations produce a planet in the HZ of their systems after the $200 \mathrm{Myr}$ of evolution. We consider the simulation labelled " 1 " as a representative of all the simulations of this scenario. Figure 10 shows snapshots of the semi-major axis-eccentricity plane of Simulation 1 , as in the previous sections. The values of the time of each panel match with the times of Figs. 3 and 6.

From Fig. 10 we can see that the inner embryos are excited and reaches eccentricities $e \leq 0.4$ at $10^{5}$ yr. However, the planetesimals show very low eccentricities with $e \leq 0.05$. At $1 \mathrm{Myr}$, most of the embryos are accreted by the surviving ones $(\sim 2 / 3$ of the missing embryos), and the rest are ejected from the system, while the planetesimals acquire higher eccentricities reaching $e \leq 0.6$. At $10 \mathrm{Myr}$ there is only one planet internal to the giant, which remains there until the end of the evolution, and four between the giant and 6 au from the central star. The number of planetesimals decreases significantly due to ejection. At 200 Myr the planet inner to the giant remains in the same spot, while only two outer planets remain. We can also see that nearly all planetesimals are removed from our studied region. In fact, $\sim 72 \%$ of the initial planetesimal population is ejected from the 

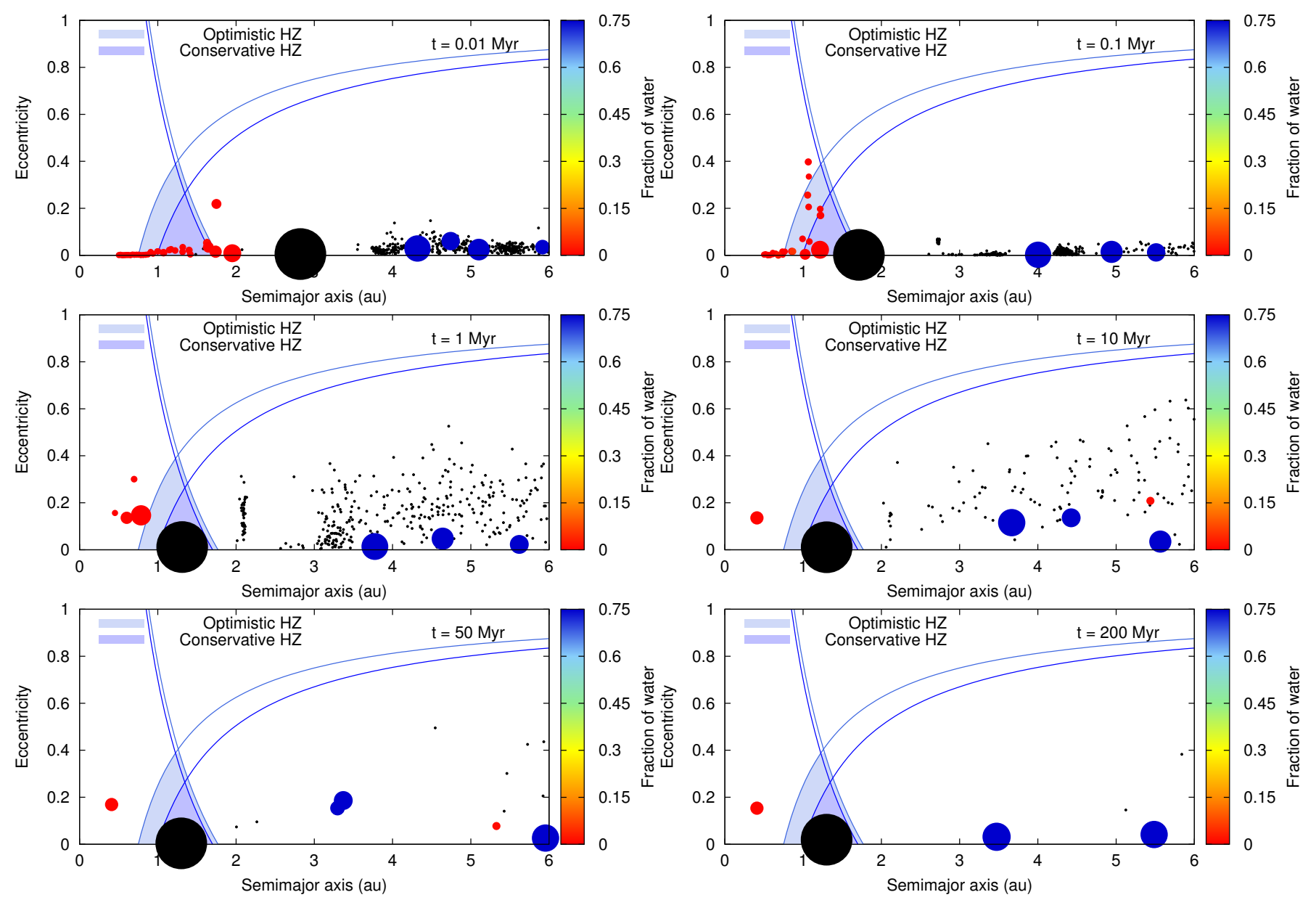

Fig. 10. Snapshots of the dynamical evolution for Simulation 1 of Scenario 3. The colour code description is analogous to that of Fig. 3.

system while only $10 \%$ remains with semi-major axes greater than 6 au.

As we see in the last panel of Fig. 10, the giant is the only planet in the HZ. Because of its position it is very difficult for another planet to survive inside the $\mathrm{HZ}$ during the evolution. To explain this, we followed the evolution of each embryo and measured its distance with respect to the giant each time it enters in the HZ. Those results yield that, when a planet is inside the HZ, the mutual distances to the giant can become as low as $\sim 0.1 R_{\text {Hill }}$, making the perturbation of the giant so large that the planet cannot survive in the HZ.

\section{Discussion and conclusions}

In this paper, we studied the main aspects concerning the planet formation in systems with a migrating gas giant around solartype stars. First of all, we used a semianalytical model to determine the parameters of the protoplanetary disk able to form a Jupiter-mass planet at the snow line. We proposed a late formation for the gaseous giant. In fact, when the giant is formed, the gaseous component remained in the system for $1.5 \times 10^{5} \mathrm{yr}$, which represented the time that the Jupiter-mass planet has to migrate. From this, we defined three scenarios of study, where the gas giant migrated up to intermediate distances of 2 au, $1.6 \mathrm{au}$, and $1.3 \mathrm{au}$ with respect to the central star. For each work scenario, the semi-analytic model also allowed us to determine the distributions of planetary embryos and planetesimals until the Jupiter-mass planet was formed. Finally, to analyse the main dynamical processes involved during and after the migration of the giant planet, we used an $N$-body code. These simulations produced a wide variety of systems, forming planets with different masses, water contents, and dynamical properties.

In particular, we were interested in studying the formation and evolution of planets in the HZ of the system. Our simulations produced three different kinds of planets in the HZ. The first kind is represented by planets whose accretion seed started the simulation beyond the snow line. Such planets ended up the simulation with significantly high water percentages by mass (up to $75 \%$ !) and owing to that, they were called water worlds. The potential habitability of the water worlds is still under debate (Abbot et al. 2012; Alibert 2014; Kitzmann et al. 2015). However, the water worlds would seem to be common in many planetary systems (de Elía et al. 2013; Ronco \& de Elía 2014) and thus, we think that they represent an interesting kind of exoplanets in the Universe. A detailed discussion about the potential habitability of water-rich planets with deep oceans can be found in Noack et al. (2016). The second kind of planets formed in the HZ is represented by those whose accretion seeds started the simulation inside the snow line and their feeding zones were primarily associated to the inner region of the disk. Such planets were more massive than the Earth and ended up the simulation with water percentages less than $\lesssim 1 \%$ by mass. The planets associated to this second kind are called Earth-like planets because they show features in their dynamical evolution and final water percentages that result to be comparable to the Earth. We think that such planets have an important astrobiological interest since they could achieve similar conditions to the Earth for the development and maintenance of life. Finally, the third kind of planets 
formed in the $\mathrm{HZ}$ is represented by those whose accretion seeds started the integration inside the snow line and did not accrete water-rich material along the evolution. These planets ended the simulation with no water and they are called dry worlds.

Those numerical simulations with a Jupiter-mass planet migrating to $\sim 2$ au produced a total number of six out of twenty planets in the HZ. In particular, two such planets were water worlds, with masses of $2.75 M_{\oplus}$ and $3.57 M_{\oplus}$ and final water contents of $75 \%$ and $58.05 \%$ by mass, respectively, which represent $\sim 7400$ Earth oceans. Within this scenario of study, the other four planets formed in the HZ were Earth-like planets. In fact, such planets were super-Earths with masses ranging from $2.15 M_{\oplus}$ to $3.85 M_{\oplus}$, and final water contents less than $0.53 \%$ by mass. Considering their masses and percentages of water, such planets showed water contents less than 73 Earth oceans. It is worth noting that we analysed the long-term dynamical stability of the resulting systems, inferring an efficient coexistence between a Jupiter-mass planet located at $\sim 2$ au and terrestrial planets in the $\mathrm{HZ}$ with a considerably wide range of masses and water contents.

The numerical simulations with a Jupiter-mass planet migrating to $\sim 1.6$ au formed a total number of three out of twenty planets in the HZ. Such planets have masses ranging from $0.58 M_{\oplus}$ to $3.51 M_{\oplus}$ and final water contents less than $1.18 \%$ by mass. Considering their masses and percentages of water, such planets showed water contents less than 36 Earth oceans. It is worth emphasizing that water worlds were not formed in this work scenario. Our analysis concerning the long-term dynamical stability of the resulting systems suggested that a Jupiter-mass planet and an Earth-like planet coexisted inside the HZ. We conclude that this result is important since it could have interesting implications in the search and discovery of exoplanets in the HZ around solar-mass stars that host a gas giant.

There are two important differences between our results and those obtained by Fogg \& Nelson (2009). The first is related to the mass of the planets surviving in the $\mathrm{HZ}$ of the systems of study. In general terms, the planets resulting from our simulations were more massive than those produced by Fogg \& Nelson (2009). In fact, on the one hand, eight of ten planets surviving in the $\mathrm{HZ}$ in our work had masses between $2.15 M_{\oplus}$ and $3.85 M_{\oplus}$. On the other hand, Fogg \& Nelson (2009) formed a planet of $2 M_{\oplus}$ in those scenarios where type I migration was excluded and migration of the giant planet was halted at distances close to the outer limit of the HZ. The second relevant difference between our work and that from Fogg \& Nelson (2009) is concerning the final water content of the planets surviving in the HZ. The planet of $2 M_{\oplus}$ produced by Fogg \& Nelson (2009) incorporated $40 \%$ of its final mass from beyond the snowline at 2.7 au. According to these authors, one effect of giant planet migration was to drive large quantities of icy material into the inner system, leading to the formation of water-rich terrestrial planets in the HZ. Even though our numerical simulations formed two true water worlds in the $\mathrm{HZ}$ of the systems of study, six of the ten planets surviving in the $\mathrm{HZ}$ showed final water contents less than $1.18 \%$ by mass. Our work suggests that this kind of planet seems to be more common than the water worlds in those scenarios where migration of the giant planet was halted close to the outer limit of the HZ.

The differences between our work and that carried out by Fogg \& Nelson (2009) are due to a combination of multiple factors. First, the mass of the protoplanetary disk used by Fogg \& Nelson (2009) was a factor of 2.2 larger than that assumed in the present work. In fact, even though both studies considered initial surface density profiles for gas and solids taken from a MMSN model (Hayashi 1981), this was scaled up in mass by factors of 1.35 and 3 for us and Fogg \& Nelson (2009), respectively. Moreover, these authors considered planetesimals of $10 \mathrm{~km}$ radius while a $0.5 M_{\text {Jup }}$ giant planet was assumed to migrate inward from 5 au. Unlike this, our work used planetesimals of 100 m radius and a Jupiter-mass planet migrated inward from $3 \mathrm{au}$. Another significant difference between our work and that from Fogg \& Nelson (2009) is related to the initial distributions of embryos and planetesimals used in the $N$-body simulations. On the one hand, Fogg \& Nelson (2009) assumed ad-hoc initial masses for the population of planetary embryos of the system. In fact, the authors chose initial masses of $0.025 M_{\oplus}$ and $0.1 M_{\oplus}$ to represent embryos interior and exterior to the snowline, respectively. Moreover, the number of planetary embryos of each $\mathrm{N}$-body simulation was calculated by the authors assuming an average radial spacing between them of eight mutual Hill radii. Unlike this, the work presented here used the semi-analytic model developed by Guilera et al. (2010) to analyse the formation of a giant planet and the evolution of embryos and planetesimals during the gaseous phase. On the other hand, Fogg \& Nelson (2009) assumed that $\sim 90 \%$ of the solid mass of the disk was contained in planetesimals. In our work, the semianalytic model computed the evolution of the planetesimal population during the gaseous phase. A more realistic treatment of the initial conditions associated to the populations of embryos and planetesimals led to more robust results concerning the final masses and water contents of the terrestrial-like planets that survived in the systems under study. Finally, it is important to remark that the $N$-body simulations developed by Fogg \& Nelson (2009) were integrated for a time span of $30 \mathrm{Myr}$, while to test the stability of the resulting systems, a few simulations were extended to $100 \mathrm{Myr}$. In the present work, we analysed the longterm dynamical stability of the systems integrating the simulations of interest for a time span of $1 \mathrm{Gyr}$.

Finally, those numerical simulations with a Jupiter-mass planet migrating to $\sim 1.3$ au did not form terrestrial planets in the HZ. In fact, the gas giant efficiently removed planetary embryos and planetesimals from that region in all our $N$-body simulations. This result is in agreement with that derived by Fogg \& Nelson (2009), who suggested that it is not conceivable that terrestrial planets survive in the HZ around a solartype star if the gaseous giant's final orbit lies inside the region of $\sim 0.3-1.5 \mathrm{au}$.

From the extrasolar systems discovered around solar-mass stars that host a Jupiter-mass planet between 1.5 au and 2 au, only two showed properties comparable to the systems of our simulations. On the one hand, HD 4208b is a gaseous giant with a minimum mass of $0.81 \pm 0.03$ Jupiter masses orbiting a central star of $0.88 M_{\odot}$ with a semi-major axis of $1.65 \pm 0.01$ au and an eccentricity of $0.05_{-0.03}^{+0.04}$ (Hollis et al. 2012). On the other hand, HD $82886 \mathrm{~b}$ is a giant planet with a minimum mass of $1.3 \pm 0.1$ Jupiter masses orbiting a central star of $1.06 \pm 0.074 M_{\odot}$ with a semi-major axis of $1.65 \pm 0.06$ au and an eccentricity less than 0.27 (Johnson et al. 2011). Although a value of 0.27 for the eccentricity of HD $82886 \mathrm{~b}$ is too large to consider it comparable to the systems of our simulations, Johnson et al. (2011) propose a possible solution which yield an eccentricity value of 0.066 . From our results, we considered that these two planetary systems could contain additional Earth-like planets in their HZs. We think that the PLATO (PLAnetary Transits and Oscillations of stars $)^{4}$ mission will be able to confirm our results by detecting

4 For more information see http://sci.esa.int/plato/ 
Earth-like planets in the HZs around Sun-like stars that host giant planets at intermediate distances.

In the three work scenarios, many $N$-body simulations produced a system of super-Earths in the outer disk, more precisely, between the location of the Jupiter-mass planet and 6 au. The gravitational microlensing technique is sensitive to planets on wide orbits. We think that the Wide-Field InfraRed Survey Telescope (WFIRST) ${ }^{5}$ mission will help to detect this kind of planets since it will, in principle, be sensitive to all planets with mass $\gtrsim 0.1 M_{\oplus}$ and separations $\gtrsim 0.5 \mathrm{AU}$, including free-floating planets.

It is important to emphasise the limitations of the semianalytic model and the $N$-body code used to carry out our study. The semi-analytic model neglects the effect of gaseous envelopes for the embryos located in the inner and outer regions respect to the position of the gas giant. However, the masses of the planetary embryos at the end of the gas phase are less than $2.7 M_{\oplus}$. Thus, we consider that such an approximation should not produce significant differences. Moreover, the semi-analytic model does not take into account the processes of planetesimal fragmentation or pebble accretion. In the same way, we did not include type I migration in our model since many quantitative aspects of such effect are still uncertain. As for the planetesimal population, the semi-analytic model assumed only one species of planetesimals with a radius of $100 \mathrm{~m}$. To consider different sizes of planetesimals, we should have produced changes in the solid surface density profile at the end of the gaseous phase. Our intention here is to describe the main simplifications of our semianalytic model. However, we consider that such a model has allowed us to carry out a good description of the evolution of the system during the gaseous phase and then, to derive more realistic conditions for the planetary embryos and the planetesimal population. To analyse the sensitivity of the results to each of the effects mentioned in the present paragraph is beyond the scope of this work.

The $N$-body MERCURY code also has some limitations that should be mentioned. On the one hand, collisions are treated as inelastic mergers, conserving mass and water content. Thus, the mass and the final water content of the resulting planets represent upper limits. Chambers (2013) developed $N$-body simulations including fragmentation and hit-and-run collisions rather than assuming that all impacts lead to a perfect merger of the colliding bodies. This improved model was based on the results of hydrodynamical simulations of planetary impacts performed by Leinhardt \& Stewart (2012) and Genda et al. (2012) that identified the boundaries of different collisional regimes and provided formulae for the mass of the largest remnant. Chambers (2013) carries out $N$-body simulations of terrestrial planet formation incorporating collisional fragmentation and hit-and-run collisions and then, he compares them to simulations in which all collisions were assumed to result in mergers. The final planetary systems formed in the two models were broadly similar. However, the author found differences concerning the planetary masses and the time-averaged eccentricities of the final planets. Future $N$-body simulations should include a more realistic treatment of the collisions in order to determine the orbital and physical characteristics of the planets in more detail. On the other hand, Marcus et al. (2010) explores the effect of late giant impacts on the final bulk abundance of water in super-Earths. According to the authors, if a giant impact between bodies of similar composition occurs, the target planet either accretes materials in the same proportion, leaving the water fraction unchanged, or loses material from the

\footnotetext{
See http://wfirst.gsfc.nasa.gov/
}

water mantle, decreasing the water fraction. Marcus et al. (2010) derives equations able to describe such processes. Other authors, such as Dvorak et al. (2015) quantify the fraction of water lost during an impact, inferring that during a collision a body might lose up to about $60 \%$ of its water content, depending on the energy and the geometry of the impact. Thus, future studies based on $N$-body simulations should include a more realistic model of the impacts in order to properly quantify the final water content. We think that the observational evidence will allow us a refinement of our models, and therefore to strengthen our understanding about the processes involved in the formation and evolution of planetary systems.

Acknowledgements. This work was partially financed by CONICET by grant PIP 0436/13. We thank the anonymous referee for valuable suggestions that helped us improve the manuscript.

\section{References}

Abbot, D. S., Cowan, N. B., \& Ciesla, F. J. 2012, ApJ, 756, 178

Adachi, I., Hayashi, C., \& Nakazawa, K. 1976, Prog. Theor. Phys., 56, 1756

Alexander, R. D., Clarke, C. J., \& Pringle, J. E. 2006, MNRAS, 369, 229

Alibert, Y. 2014, A\&A, 561, A41

Alibert, Y., Mousis, O., Mordasini, C., \& Benz, W. 2005, ApJ, 626, L57

Armitage, P. J. 2003, ApJ, 582, L47

Armitage, P. J. 2010, Astrophysics of Planet Formation (Cambridge, UK: Cambridge University Press), 294

Benítez-Llambay, P., Masset, F., Koenigsberger, G., \& Szulágyi, J. 2015, Nature, 520, 63

Bibring, J.-P., Langevin, Y., Mustard, J. F., et al. 2006, Science, 312, 400

Chambers, J. E. 1999, MNRAS, 304, 793

Chambers, J. 2008, Icarus, 198, 256

Chambers, J. E. 2013, Icarus, 224, 43

Chiang, E. I., Fischer, D., \& Thommes, E. 2002, ApJ, 564, L105

Cresswell, P., \& Nelson, R. P. 2008, A\&A, 482, 677

Cumming, A., Butler, R. P., Marcy, G. W., et al. 2008, PASP, 120, 531

Dauphas, N., \& Pourmand, A. 2011, Nature, 473, 489

de Elía, G. C., Guilera, O. M., \& Brunini, A. 2013, A\&A, 557, A42

Dittkrist, K.-M., Mordasini, C., Klahr, H., Alibert, Y., \& Henning, T. 2014, A\&A, 567, A121

Dvorak, R., Maindl, T. I., Burger, C., et al. 2015, Nonlinear Phenomena in Complex Systems, 18, 310

Fogg, M. J., \& Nelson, R. P. 2005, A\&A, 441, 791

Fogg, M. J., \& Nelson, R. P. 2007, A\&A, 461, 1195

Fogg, M. J., \& Nelson, R. P. 2009, A\&A, 498, 575

Fortier, A., Benvenuto, O. G., \& Brunini, A. 2009, A\&A, 500, 1249

Genda, H., Kokubo, E., \& Ida, S. 2012, ApJ, 744, 137

Guilera, O. M., Brunini, A., \& Benvenuto, O. G. 2010, A\&A, 521, A50

Guilera, O. M., Fortier, A., Brunini, A., \& Benvenuto, O. G. 2011, A\&A, 532, A142

Guilera, O. M., de Elía, G. C., Brunini, A., \& Santamaría, P. J. 2014, A\&A, 565, A96

Guilet, J., Baruteau, C., \& Papaloizou, J. C. B. 2013, MNRAS, 430, 1764

Hayashi, C. 1981, Prog. Theor. Phys. Suppl., 70, 35

Hollis, M. D. J., Balan, S. T., Lever, G., \& Lahav, O. 2012, MNRAS, 423, 2800 Howard, A. W. 2013, Science, 340, 572

Hubickyj, O., Bodenheimer, P., \& Lissauer, J. J. 2005, Icarus, 179, 415

Ida, S., \& Lin, D. N. C. 2008, ApJ, 673, 487

Ida, S., \& Makino, J. 1993, Icarus, 106, 210

Ida, S., Lin, D. N. C., \& Nagasawa, M. 2013, ApJ, 775, 42

Inaba, S., Tanaka, H., Nakazawa, K., Wetherill, G. W., \& Kokubo, E. 2001, Icarus, 149, 235

Jacobson, S. A., Morbidelli, A., Raymond, S. N., et al. 2014, Nature, 508, 84

Johnson, J. A., Clanton, C., Howard, A. W., et al. 2011, ApJS, 197, 26

Kitzmann, D., Alibert, Y., Godolt, M., et al. 2015, MNRAS, 452, 3752

Kley, W., \& Crida, A. 2008, A\&A, 487, L9

Kopparapu, R. K., Ramirez, R., Kasting, J. F., et al. 2013a, ApJ, 770, 82

Kopparapu, R. K., Ramirez, R., Kasting, J. F., et al. 2013b, ApJ, 765, 131

Kuchner, M. J. 2003, ApJ, 596, L105

Léger, A., Selsis, F., Sotin, C., et al. 2004, Icarus, 169, 499

Leinhardt, Z. M., \& Stewart, S. T. 2012, ApJ, 745, 79

Lin, D. N. C., Bodenheimer, P., \& Richardson, D. C. 1996, Nature, 380, 606

Mamajek, E. E. 2009, in AIP Conf. Ser., eds. T. Usuda, M. Tamura, \& M. Ishii, 1158,3

Mandell, A. M., \& Sigurdsson, S. 2003, ApJ, 599, L111 
Mandell, A. M., Raymond, S. N., \& Sigurdsson, S. 2007, ApJ, 660, 823

Marcus, R. A., Sasselov, D., Stewart, S. T., \& Hernquist, L. 2010, ApJ, 719, L45

Marty, B. 2012, Earth Planet. Sci. Lett., 313, 56

Masset, F. S., D’Angelo, G., \& Kley, W. 2006, ApJ, 652, 730

Miguel, Y., Guilera, O. M., \& Brunini, A. 2011a, MNRAS, 417, 314

Miguel, Y., Guilera, O. M., \& Brunini, A. 2011b, MNRAS, 412, 2113

Mordasini, C. 2014, A\&A, 572, A118

Mordasini, C., Alibert, Y., \& Benz, W. 2009, A\&A, 501, 1139

Noack, L., Höning, D., Rivoldini, A., et al. 2016, Icarus, 277, 215

O’Brien, D. P., Morbidelli, A., \& Levison, H. F. 2006, Icarus, 184, 39

Ohtsuki, K., Stewart, G. R., \& Ida, S. 2002, Icarus, 155, 436

Ormel, C. W. 2014, ApJ, 789, L18

Paardekooper, S.-J., Baruteau, C., Crida, A., \& Kley, W. 2010, MNRAS, 401, 1950

Paardekooper, S.-J., Baruteau, C., \& Kley, W. 2011, MNRAS, 410, 293

Pollack, J. B., Kasting, J. F., Richardson, S. M., \& Poliakoff, K. 1987, Icarus, 71, 203
Pollack, J. B., Hubickyj, O., Bodenheimer, P., et al. 1996, Icarus, 124, 62 Rafikov, R. R. 2004, AJ, 128, 1348

Raymond, S. N., Quinn, T., \& Lunine, J. I. 2004, Icarus, 168, 1

Raymond, S. N., Quinn, T., \& Lunine, J. I. 2006, Icarus, 183, 265

Raymond, S. N., O’Brien, D. P., Morbidelli, A., \& Kaib, N. A. 2009, Icarus, 203 644

Ronco, M. P., \& de Elía, G. C. 2014, A\&A, 567, A54

Ronco, M. P., Guilera, O. M., \& de Elía, G. C. 2017, MNRAS, 471, 2753

Shakura, N. I., \& Sunyaev, R. A. 1973, A\&A, 24, 337

Solomon, S. C., \& Head, J. W. 1991, Science, 252, 252

Tanaka, H., \& Ida, S. 1999, Icarus, 139, 350

Tanaka, H., Takeuchi, T., \& Ward, W. R. 2002, ApJ, 565, 1257

Touboul, M., Kleine, T., Bourdon, B., Palme, H., \& Wieler, R. 2007, Nature, 450, 1206

Walsh, K. J., \& Morbidelli, A. 2011, A\&A, 526, A126

Williams, D. M., \& Pollard, D. 2002, Int. J. Astrobiol., 1, 61 